\title{
Self-adjointness of the two-dimensional massless Dirac Hamiltonian and vacuum polarization effects in the background of a singular magnetic vortex
}

\author{
Yurii A. Sitenko* \\ Bogolyubov Institute for Theoretical Physics, National Academy of Sciences, \\ 14-b Metrologichna str., 03143 Kyiv, Ukraine ${ }^{\dagger}$ \\ and \\ Institute for Theoretical Physics, Berne University, \\ Sidlerstrasse 5, 3012 Berne, Switzerland
}

\begin{abstract}
A massless spinor field is quantized in the background of a singular static magnetic vortex in 2+1-dimensional space-time. The method of self-adjoint extensions is employed to define the most general set of physically acceptable boundary conditions at the location of the vortex. Under these conditions, all effects of polarization of the massless fermionic vacuum in the vortex background are determined. Absence of anomaly is demonstrated, and patterns of both parity and chiral symmetry breaking are discussed.
\end{abstract}

PACS number(s): 03.65.Bz, 12.20.Ds, 11.30.Er, 11.30.Qc

Keywords: vacuum polarization, singular vortex, self-adjointness

\section{INTRODUCTION}

Singular (or contact or zero-range) interaction potentials were introduced in quantum mechanics more than sixty years ago 11 3]. Since that time the attitude of physicists and mathematicians to this subject was varying, starting from "it is impossible", then to "it is evident", and finally arriving at "it is interesting" (for a review see monograph [4]). A mathematically consistent and rigorous treatment of the subject was developed [5], basing on the notion of self-adjoint extension of a Hermitian (symmetric) operator.

Singular interaction is involved in quantum field theory when, for example, a spinor field is quantized in the background of a pointlike magnetic monopole in threedimensional space or a pointlike magnetic vortex in twodimensional space. In these cases the Dirac Hamiltonian, in contrast to the Schrodinger one, is free from an explicit $\delta$-function singularity; nonetheless the problem of self-adjoint extension of both Dirac and Schrodinger operators arises, albeit for different reasons (see, for example [6]). A distinguishing feature is that a solution to the Dirac equation, unlike that to the Schrodinger one, cannot obey a condition of regularity at the singularity point. It is necessary then to define a boundary condition at this point, and the least restrictive, but still physically acceptable, condition is such that guarantees self-adjointness of the Dirac Hamiltonian. Thus, effects of polarization of the fermionic vacuum in a singular background (such as a pointlike monopole or a pointlike vortex) appear to depend on the choice of the boundary condition at the singularity point, and a set of permissible boundary conditions is labelled, most generally, by the values of self-adjoint extension parameters. In contrast to the Schrodinger case, the extension in the Dirac case does not reflect additional types of interaction but represents complementary information that must be specified when describing the physical attributes of the already posited singular background configuration.

As a consequence, the fermionic vacuum under the influence of a singular background can acquire rather unusual properties: leakage of quantum numbers from the singularity point occurs. While in the case of a monopole there is

\footnotetext{
*Electronic address: yusitenko@bitp.kiev.ua

${ }^{\dagger}$ Permanent address
} 
leakage of charge to the vacuum, which results in the monopole becoming the dyon violating the Dirac quantization condition and CP symmetry [7] 11], in the case of a vortex (the Ehrenberg-Siday-Aharonov-Bohm potential [12, 13]) the situation is much more complicated, since there is leakage of both charge and other quantum numbers to the vacuum. Apparently, this is due to a nontrivial topology of the base space in the latter case: $\pi_{1}=0$ in the case of a space with a deleted point, and $\pi_{1}=\mathbb{Z}$ in the case of a space with a deleted line (or a plane with a deleted point); here $\pi_{1}$ is the first homotopy group and $\mathbb{Z}$ is a set of integer numbers. For a particular choice of the boundary condition at the location of a singular vortex it has been shown that charge [14,15], current [16] and angular momentum [17] are induced in the vacuum. The induced vacuum quantum numbers under general boundary conditions which are compatible with self-adjointness have been considered in Refs. 18 21].

Thus far the effects of polarization of the massive fermionic vacuum have been studied. In a 2+1-dimensional space-time the mass term for a spinor field in an irreducible representation of the Clifford algebra violates both types of parity - under space and time reflections. One can also consider a third type of parity, which is similar to the axial symmetry in evendimensional space-times (see Refs. 222 25]) and which is violated by the mass term as well.

When quantization of a massless spinor field is considered, all above symmetries are formally present. Our concern is then, in the first place, in the following: whether the vacuum polarization effects in a singular background respect these formal symmetries? It will be shown that, although the parity anomaly is absent, the parity breaking condensate emerges in the vacuum. If the number of quantized spinor fields is doubled to form a reducible representation composed of two inequivalent irreducible ones, then this condensate becomes parity conserving but chiral symmetry breaking. Also all other characteristics of the massless fermionic vacuum in a singular background are determined.

A singular background in $2+1$ dimensional space-time is taken in the form of a pointlike static magnetic vortex (the Ehrenberg-Siday-Aharonov-Bohm configuration)

$$
\begin{gathered}
V^{1}(\mathbf{x})=-\Phi^{(0)} \frac{x^{2}}{\left(x^{1}\right)^{2}+\left(x^{2}\right)^{2}}, \quad V^{2}(\mathbf{x})=\Phi^{(0)} \frac{x^{1}}{\left(x^{1}\right)^{2}+\left(x^{2}\right)^{2}}, \\
\boldsymbol{\partial} \times \mathbf{V}(\mathbf{x})=2 \pi \Phi^{(0)} \delta(\mathbf{x}),
\end{gathered}
$$

where $\Phi^{(0)}$ is the vortex flux in $2 \pi$ units, i.e. in the London $\left(2 \pi \hbar c e^{-1}\right)$ units, since we use conventional units $\hbar=c=1$ and coupling constant $e$ is included into vector potential $\mathbf{V}(\mathbf{x})$. The wave function on the plane $\left(x^{1}, x^{2}\right)$ with punctured singular point $x^{1}=x^{2}=0$ obeys the most general condition (see [18] for more details)

$$
<r, \varphi+2 \pi\left|=e^{i 2 \pi \Upsilon}<r, \varphi\right|,
$$

where $r=\sqrt{\left(x^{1}\right)^{2}+\left(x^{2}\right)^{2}}$ and $\varphi=\arctan \left(x^{2} / x^{1}\right)$ are the polar coordinates, and $\Upsilon$ is a continuous real parameter which is varied in the range $0 \leq \Upsilon<1$. It can be shown (see, for example, 17, 18]) that $\Upsilon$ as well as $\Phi^{(0)}$ is changed under singular gauge transformations, whereas difference $\Phi^{(0)}-\Upsilon$ remains invariant. Thus, physically sensible quantities are to depend on the gauge invariant combination $\Phi^{(0)}-\Upsilon$ which will be for brevity denoted as the reduced vortex flux in the following.

In the next section, the most general set of physically acceptable boundary conditions at the singularity point $\mathbf{x}=0$ is defined. In Section III, the vacuum fermion number is determined. Section IV is central from the technical point of view, since we show here, how the problem of both ultraviolet and infrared divergences in vacuum characteristics is solved with the help of zeta function regularization. This allows us to get immediately in Section V the vacuum energy density; also here the effective action and the effective potential are considered. The vacuum current is determined in Section VI, and the vacuum condensate is determined in Section VII. We demonstrate in Section VIII that the parity anomaly is absent in the background of a singular magnetic vortex. Section IX is devoted to the determination of the last vacuum characteristics - angular momentum. We summarize results and discuss their consequences in Section $\mathrm{X}$. Some crucial points of the derivation of results are outlined in Appendices A-D. 


\section{QUANTIZATION OF A SPINOR FIELD AND THE BOUNDARY CONDITION AT THE LOCATION OF A VORTEX}

The operator of the second-quantized spinor field is presented in the form

$$
\Psi(\mathbf{x}, t)=\sum_{E_{\lambda}>0} e^{-i E_{\lambda} t}<\mathbf{x}\left|\lambda>a_{\lambda}+\sum_{E_{\lambda}<0} e^{-i E_{\lambda} t}<\mathbf{x}\right| \lambda>b_{\lambda}^{+},
$$

where $a_{\lambda}^{+}$and $a_{\lambda}\left(b_{\lambda}^{+}\right.$and $\left.b_{\lambda}\right)$ are the spinor particle (antiparticle) creation and annihilation operators satisfying anticommutation relations

$$
\left[a_{\lambda}, a_{\lambda^{\prime}}^{+}\right]_{+}=\left[b_{\lambda}, b_{\lambda^{\prime}}^{+}\right]_{+}=<\lambda \mid \lambda^{\prime}>
$$

and $\langle\mathbf{x} \mid \lambda\rangle$ is the solution to the stationary Dirac equation

$$
H<\mathbf{x}\left|\lambda>=E_{\lambda}<\mathbf{x}\right| \lambda>
$$

$H$ is the Dirac Hamiltonian, $\lambda$ is the set of parameters (quantum numbers) specifying a state, $E_{\lambda}$ is the energy of a

state; symbol $\mathcal{Y}$ means the summation over discrete and the integration (with a certain measure) over continuous values of $\lambda$. The ground state $\mid \operatorname{vac}>$ is defined conventionally by equality

$$
a_{\lambda}\left|\operatorname{vac}>=b_{\lambda}\right| \operatorname{vac}>=0 .
$$

In the case of quantization of a massless spinor field in the background of static vector field $\mathbf{V}(\mathbf{x})$, the Dirac Hamiltonian takes the form

$$
H=-i \boldsymbol{\alpha}[\boldsymbol{\partial}-i \mathbf{V}(\mathbf{x})]
$$

where

$$
\boldsymbol{\alpha}=\gamma^{0} \gamma, \quad \beta=\gamma^{0},
$$

$\gamma^{0}$ and $\gamma$ are the Dirac $\gamma$ matrices. In the 2+1-dimensional space-time $(\mathbf{x}, t)=\left(x^{1}, x^{2}, t\right)$ the Clifford algebra has two inequivalent irreducible representations which can be differed in the following way:

$$
i \gamma^{0} \gamma^{1} \gamma^{2}=s, \quad s= \pm 1
$$

Choosing the $\gamma^{0}$ matrix in the diagonal form

$$
\gamma^{0}=\sigma_{3}
$$

one gets

$$
\gamma^{1}=e^{\frac{i}{2} \sigma_{3} \chi_{s}} i \sigma_{1} e^{-\frac{i}{2} \sigma_{3} \chi_{s}}, \quad \gamma^{2}=e^{\frac{i}{2} \sigma_{3} \chi_{s}} i s \sigma_{2} e^{-\frac{i}{2} \sigma_{3} \chi_{s}},
$$

where $\sigma_{1}, \sigma_{2}$ and $\sigma_{3}$ are the Pauli matrices, and $\chi_{1}$ and $\chi_{-1}$ are the parameters that are varied in the interval $0 \leq \chi_{s}<2 \pi$ to go over to the equivalent representations.

A solution to the Dirac equation (2.3) with Hamiltonian (2.5) in background (1.1), that obeys condition (1.3), can be presented as

$$
<\mathbf{x} \mid E, n>=\left(\begin{array}{l}
f_{n}(r, E) e^{i(n+\Upsilon) \varphi} \\
g_{n}(r, E) e^{i(n+\Upsilon+s) \varphi}
\end{array}\right), \quad n \in \mathbb{Z},
$$


where the column of radial functions $\left(\begin{array}{c}f_{n} \\ g_{n}\end{array}\right)$ satisfies the equation

$$
h_{n}\left(\begin{array}{c}
f_{n} \\
g_{n}
\end{array}\right)=E\left(\begin{array}{l}
f_{n} \\
g_{n}
\end{array}\right)
$$

and

$$
h_{n}=\left(\begin{array}{cc}
0 & e^{i \chi_{s}}\left[\partial_{r}+s\left(n-\Phi^{(0)}+\Upsilon+s\right) r^{-1}\right] \\
e^{-i \chi_{s}}\left[-\partial_{r}+s\left(n-\Phi^{(0)}+\Upsilon\right) r^{-1}\right] & 0
\end{array}\right)
$$

is the partial Dirac Hamiltonian. When reduced vortex flux $\Phi^{(0)}-\Upsilon$ is integer, the requirement of square integrability for wave function (2.10) at $r \rightarrow 0$ provides its regularity, rendering the partial Dirac Hamiltonian $h_{n}$ for every value of $n$ to be essentially self-adjoint. When $\Phi^{(0)}-\Upsilon$ is fractional, the same is valid only for $n \neq n_{0}$, where

$$
n_{0}=\left[\left[\Phi^{(0)}-\Upsilon\right]\right]+\frac{1}{2}-\frac{1}{2} s
$$

$[[u]]$ is the integer part of a quantity $u$ (i.e., the greatest integer that is less than or equal to $u$ ). For $n=n_{0}$, each of the two linearly independent solutions to Eq.(2.11) meets the requirement of square integrability at $r \rightarrow 0$. Any particular solution in this case is characterized by at least one (at most both) of the radial functions being divergent as $r^{-p}$ $(p<1)$ at $r \rightarrow 0$. If one of the two linearly independent solutions is chosen to have a regular upper and an irregular lower component, then the other one has a regular lower and an irregular upper component. Therefore, in contrast to operator $h_{n}\left(n \neq n_{0}\right)$, operator $h_{n_{0}}$ is not essentially self-adjoint . The Weyl - von Neumann theory of self-adjoint operators (see, e.g., Refs. [4 27) has to be employed in order to consider the possibility of a self-adjoint extension in the case of $n=n_{0}$. It is shown in Appendix A that the self-adjoint extension exists indeed and is parametrized by one continuous real variable denoted in the following by $\Theta$. Thus operator $h_{n_{0}}$ is defined on the domain of functions obeying the condition

$$
\cos \left(s \frac{\Theta}{2}+\frac{\pi}{4}\right) \lim _{r \rightarrow 0}(\mu r)^{F} f_{n_{0}}=-e^{i \chi_{s}} \sin \left(s \frac{\Theta}{2}+\frac{\pi}{4}\right) \lim _{r \rightarrow 0}(\mu r)^{1-F} g_{n_{0}},
$$

where $\mu>0$ is the parameter of the dimension of inverse length and

$$
F=s\left\{\left|\Phi^{(0)}-\Upsilon\right|\right\}+\frac{1}{2}-\frac{1}{2} s,
$$

$\{|u|\}=u-[[u]]$ is the fractional part of a quantity $u, 0 \leq\{\mid u\}<1$; note here that Eq.(2.14) implies that $0<F<1$, since in the case of $F=\frac{1}{2}-\frac{1}{2} s$ both $f_{n_{0}}$ and $g_{n_{0}}$ obey the condition of regularity at $r \rightarrow 0$. Note also that Eq.(2.14) is periodic in $\Theta$ with period $2 \pi$; therefore, without a loss of generality, all permissible values of $\Theta$ will be restricted in the following to range $-\pi \leq \Theta \leq \pi$.

All solutions to the massless Dirac equation in the background of a singular magnetic vortex correspond to the continuous spectrum and, therefore, obey the orthonormality condition

$$
\int d^{2} x<E, n|\mathbf{x}><\mathbf{x}| E^{\prime}, n^{\prime}>=\frac{\delta\left(E-E^{\prime}\right)}{\sqrt{\left|E E^{\prime}\right|}} \delta_{n n^{\prime}}
$$

\footnotetext{
${ }^{1}$ A corollary of the theorem proven in Ref. 26 states that, for the partial Dirac Hamiltonian to be essentially self-adjoint, it is necessary and sufficient that a non-square-integrable (at $r \rightarrow 0$ ) solution exist.
} 
In the case of $0<F<1$ one can get the following expressions corresponding to the regular solutions with $s n>s n_{0}$ :

$$
\left(\begin{array}{l}
f_{n} \\
g_{n}
\end{array}\right)=\frac{1}{2 \sqrt{\pi}}\left(\begin{array}{c}
J_{l-F}(k r) e^{i \chi_{s}} \\
\operatorname{sgn}(E) J_{l+1-F}(k r)
\end{array}\right), \quad l=s\left(n-n_{0}\right),
$$

the regular solutions with $s n<s n_{0}$ :

$$
\left(\begin{array}{l}
f_{n} \\
g_{n}
\end{array}\right)=\frac{1}{2 \sqrt{\pi}}\left(\begin{array}{c}
J_{l^{\prime}+F}(k r) e^{i \chi_{s}} \\
-\operatorname{sgn}(E) J_{l^{\prime}-1+F}(k r)
\end{array}\right), \quad l^{\prime}=s\left(n_{0}-n\right),
$$

and the irregular solution:

$$
\left(\begin{array}{c}
f_{n_{0}} \\
g_{n_{0}}
\end{array}\right)=\frac{1}{2 \sqrt{\pi\left[1+\sin \left(2 \nu_{E}\right) \cos (F \pi)\right]}}\left(\begin{array}{c}
{\left[\sin \left(\nu_{E}\right) J_{-F}(k r)+\cos \left(\nu_{E}\right) J_{F}(k r)\right] e^{i \chi_{s}}} \\
\operatorname{sgn}(E)\left[\sin \left(\nu_{E}\right) J_{1-F}(k r)-\cos \left(\nu_{E}\right) J_{-1+F}(k r)\right]
\end{array}\right) ;
$$

here $k=|E|, J_{\rho}(u)$ is the Bessel function of order $\rho$ and

$$
\operatorname{sgn}(u)=\left\{\begin{array}{cc}
1, & u>0 \\
-1, & u<0
\end{array}\right\}
$$

Substituting the asymptotic form of Eq.(2.19) at $r \rightarrow 0$ into Eq.(2.14), one arrives at the relation between parameters $\nu_{E}$ and $\Theta$ :

$$
\tan \left(\nu_{E}\right)=\operatorname{sgn}(E)\left(\frac{k}{2 \mu}\right)^{2 F-1} \frac{\Gamma(1-F)}{\Gamma(F)} \tan \left(s \frac{\Theta}{2}+\frac{\pi}{4}\right)
$$

where $\Gamma(u)$ is the Euler gamma function.

Using the explicit form of solutions $(2.17)$ - (2.19), all vacuum polarization effects can be determined.

\section{FERMION NUMBER}

In the second-quantized theory in 2+1-dimensional space-time the operator of the fermion number is given by the expression

$$
\hat{\mathcal{N}}=\int d^{2} x \frac{1}{2}\left[\Psi^{+}(\mathbf{x}, t), \Psi(\mathbf{x}, t)\right]_{-}=\bigvee\left[a_{\lambda}^{+} a_{\lambda}-b_{\lambda}^{+} b_{\lambda}-\frac{1}{2} \operatorname{sgn}\left(E_{\lambda}\right)\right]
$$

and, consequently, its vacuum expectation value takes the form

$$
\mathcal{N} \equiv<\operatorname{vac}|\hat{\mathcal{N}}| \operatorname{vac}>=-\frac{1}{2} \sum \operatorname{sgn}\left(E_{\lambda}\right)=-\frac{1}{2} \int d^{2} x \operatorname{tr}<\mathbf{x}|\operatorname{sgn}(H)| \mathbf{x}>.
$$

From general arguments, one could expect that the last quantity vanishes due to cancellation between the contributions of positive and negative energy solutions to the Dirac equation (2.3). Namely this happens in a lot of cases. That is why every case of a nonvanishing value of $\mathcal{N}$ deserves a special attention.

Considering the case of the background in the form of a singular magnetic vortex (1.1) - (1.2), one can notice that the contribution of regular solutions (2.17) and (2.18) is cancelled upon summation over the sign of energy, whereas irregular solution (2.19) yields a nonvanishing contribution to $\mathcal{N}(3.2)$. Defining the vacuum fermion number density

$$
\mathcal{N}_{\mathbf{x}}=-\frac{1}{2} \operatorname{tr}<\mathbf{x}|\operatorname{sgn}(H)| \mathbf{x}>
$$


we get

$$
\begin{aligned}
\mathcal{N}_{\mathbf{x}}= & -\frac{1}{8 \pi} \int_{0}^{\infty} d k k\left\{A\left(\frac{k}{\mu}\right)^{2 F-1}\left[L_{(+)}+L_{(-)}\right]\left[J_{-F}^{2}(k r)+J_{1-F}^{2}(k r)\right]+\right. \\
& +2\left[L_{(+)}-L_{(-)}\right]\left[J_{-F}(k r) J_{F}(k r)-J_{1-F}(k r) J_{-1+F}(k r)\right]+ \\
& \left.+A^{-1}\left(\frac{k}{\mu}\right)^{1-2 F}\left[L_{(+)}+L_{(-)}\right]\left[J_{F}^{2}(k r)+J_{-1+F}^{2}(k r)\right]\right\},
\end{aligned}
$$

where

$$
A=2^{1-2 F} \frac{\Gamma(1-F)}{\Gamma(F)} \tan \left(s \frac{\Theta}{2}+\frac{\pi}{4}\right)
$$

and

$$
L_{( \pm)}=2^{-1}\left\{\cos (F \pi) \pm \cosh \left[(2 F-1) \ln \left(\frac{k}{\mu}\right)+\ln A\right]\right\}^{-1} .
$$

We show in Appendix B, how the integral in Eq.(3.4) is transformed resulting in the expression

$$
\mathcal{N}_{\mathbf{x}}=-\frac{\sin (F \pi)}{2 \pi^{3} r^{2}} \int_{0}^{\infty} d w w \frac{K_{F}^{2}(w)-K_{1-F}^{2}(w)}{\cosh \left[(2 F-1) \ln \left(\frac{w}{\mu r}\right)+\ln A\right]},
$$

where $K_{\rho}(w)$ is the Macdonald function of order $\rho$. Vacuum fermion number density (3.7) vanishes at half-integer values of reduced vortex flux $\Phi^{(0)}-\Upsilon$ (i.e. at $F=\frac{1}{2}$ ) as well as at $\cos \Theta=0$. Otherwise, at large distances from the vortex we get

$$
\mathcal{N}_{\mathbf{x}_{r \rightarrow \infty}}-\left(F-\frac{1}{2}\right) \frac{\sin (F \pi)}{2 \pi^{2} r^{2}}\left\{\begin{array}{cl}
(\mu r)^{2 F-1} A^{-1} \frac{\Gamma\left(\frac{3}{2}-F\right) \Gamma\left(\frac{3}{2}-2 F\right)}{\Gamma(2-F)}, & 0<F<\frac{1}{2} \\
(\mu r)^{1-2 F} A \frac{\Gamma\left(F+\frac{1}{2}\right) \Gamma\left(2 F-\frac{1}{2}\right)}{\Gamma(1+F)}, & \frac{1}{2}<F<1
\end{array} .\right.
$$

Integrating Eq.(3.7) over the plane $\left(x^{1}, x^{2}\right)$, we obtain the total vacuum fermion number

$$
\mathcal{N}=-\frac{1}{2} \operatorname{sgn}_{0}\left[\left(F-\frac{1}{2}\right) \cos \Theta\right]
$$

where

$$
\operatorname{sgn}_{0}(u)=\left\{\begin{array}{cc}
\operatorname{sgn}(u), & u \neq 0 \\
0, & u=0
\end{array}\right\}
$$

\section{ZETA FUNCTION}

In the second-quantized theory the operator of energy is defined as

$$
\hat{\mathcal{E}}=\int d^{2} x \frac{1}{2}\left[\Psi^{+}(\mathbf{x}, t), H \Psi(\mathbf{x}, t)\right]_{-}=\oint\left(E_{\lambda} a_{\lambda}^{+} a_{\lambda}-E_{\lambda} b_{\lambda}^{+} b_{\lambda}-\frac{1}{2}\left|E_{\lambda}\right|\right),
$$

thus the vacuum expectation value of the energy takes the form 


$$
\mathcal{E} \equiv<\operatorname{vac}|\hat{\mathcal{E}}| \operatorname{vac}>=-\frac{1}{2} \bigvee\left|E_{\lambda}\right|=-\frac{1}{2} \int d^{2} x \operatorname{tr}<\mathbf{x}|| H|| \mathbf{x}>
$$

The latter expression is ill-defined due to divergences of various kinds. First, there is a bulk divergence resulting from the integration over the infinite twodimensional space. But, even if one considers the vacuum energy density,

$$
\mathcal{E}_{\mathbf{x}}=-\frac{1}{2} \operatorname{tr}\langle\mathbf{x}|| H|| \mathbf{x}\rangle
$$

still it remains to be divergent. There is a divergence at large values of momentum of integration, $k \rightarrow \infty$. To tame this divergence, let us introduce the zeta function density

$$
\zeta_{\mathbf{x}}(z)=\operatorname{tr}<\left.\mathbf{x}|| H\right|^{-2 z} \mid \mathbf{x}>
$$

which is ultraviolet convergent at sufficiently large values of $\operatorname{Re} z$. However, exactly at these values of $\operatorname{Re} z$ the integral corresponding to Eq.(4.4) is divergent in the infrared region, as $k \rightarrow 0$. To regularize this last divergence, let us introduce fermion mass $m$, modifying definition (4.4):

$$
\zeta_{\mathbf{x}}(z \mid m)=\operatorname{tr}<\left.\mathbf{x}|| \tilde{H}\right|^{-2 z} \mid \mathbf{x}>
$$

where

$$
\tilde{H}=-i \boldsymbol{\alpha}[\boldsymbol{\partial}-i \mathbf{V}(\mathbf{x})]+\beta m,
$$

and it is implied that the complete set of solutions to the equation

$$
\tilde{H}<\mathbf{x}\left|\lambda>=\tilde{E}_{\lambda}<\mathbf{x}\right| \lambda>,
$$

instead of those to Eq.(2.3), is used.

In the background of a singular magnetic vortex (1.1) - (1.2) the radial functions of the solutions to Eq.(4.7) take the form:

$$
\begin{gathered}
\left(\begin{array}{c}
\tilde{f}_{n} \\
\tilde{g}_{n}
\end{array}\right)=\frac{1}{2 \sqrt{\pi}}\left(\begin{array}{c}
\sqrt{1+m \tilde{E}^{-1}} J_{l-F}(k r) e^{i \chi_{s}} \\
\operatorname{sgn}(\tilde{E}) \sqrt{1-m \tilde{E}^{-1}} J_{l+1-F}(k r)
\end{array}\right), \quad l=s\left(n-n_{0}\right)>0 \\
\left(\begin{array}{c}
\tilde{f}_{n} \\
\tilde{g}_{n}
\end{array}\right)=\frac{1}{2 \sqrt{\pi}}\left(\begin{array}{c}
\sqrt{1+m \tilde{E}^{-1}} J_{l^{\prime}+F}(k r) e^{i \chi_{s}} \\
-\operatorname{sgn}(\tilde{E}) \sqrt{1-m \tilde{E}^{-1}} J_{l^{\prime}-1+F}(k r)
\end{array}\right), \quad l^{\prime}=s\left(n_{0}-n\right)>0 \\
\left(\begin{array}{c}
\tilde{f}_{n_{0}}^{(C)} \\
\tilde{g}_{n_{0}}^{(C)}
\end{array}\right)=\frac{1}{2 \sqrt{\pi\left[1+\sin \left(2 \tilde{\nu}_{\tilde{E}}\right) \cos (F \pi)\right.}} \times \\
\times\left(\begin{array}{c}
\sqrt{1+m \tilde{E}^{-1}}\left[\sin \left(\tilde{\nu}_{\tilde{E}}\right) J_{-F}(k r)+\cos \left(\tilde{\nu}_{\tilde{E}}\right) J_{F}(k r)\right] e^{i \chi_{s}} \\
\operatorname{sgn}(\tilde{E}) \sqrt{1-m \tilde{E}^{-1}}\left[\sin \left(\tilde{\nu}_{\tilde{E}}\right) J_{1-F}(k r)-\cos \left(\tilde{\nu}_{\tilde{E}}\right) J_{-1+F}(k r)\right]
\end{array}\right)
\end{gathered}
$$


where $k=\sqrt{\tilde{E}^{2}-m^{2}}$,

$$
\tan \left(\tilde{\nu}_{\tilde{E}}\right)=\operatorname{sgn}(\tilde{E}) \sqrt{\frac{1-m \tilde{E}^{-1}}{1+m \tilde{E}^{-1}}}\left(\frac{k}{\mu}\right)^{2 F-1} A
$$

$A$ is given by Eq.(3.5); note that the radial functions of irregular solution (4.10) satisfy condition (2.14) (see Appendix A). Note also that Eqs.(4.8) - (4.10) correspond to the continuum, $|\tilde{E}|>|m|$ 2. In addition to them, in the case of

$$
\operatorname{sgn}(m) \cos \Theta<0
$$

an irregular solution corresponding to the bound state appears. Its radial functions are

$$
\left(\begin{array}{l}
\tilde{f}_{n_{0}}^{(\mathrm{BS})} \\
\tilde{g}_{n_{0}}^{(\mathrm{BS})}
\end{array}\right)=\frac{\kappa}{\pi} \sqrt{\frac{\sin (F \pi)}{1+(2 F-1) m^{-1} E_{\mathrm{BS}}}}\left(\begin{array}{c}
\sqrt{1+m^{-1} E_{\mathrm{BS}}} K_{F}(\kappa r) e^{i \chi_{s}} \\
\left.\operatorname{sgn}(m) \sqrt{1-m^{-1} E_{\mathrm{BS}}} K_{1-F}(\kappa r)\right]
\end{array}\right),
$$

where $\kappa=\sqrt{m^{2}-E_{\mathrm{BS}}^{2}}$ and the bound state energy $\tilde{E}=E_{\mathrm{BS}}\left(\left|E_{\mathrm{BS}}\right|<|m|\right)$ is determined implicitly by the equation

$$
\frac{\left(1+m^{-1} E_{\mathrm{BS}}\right)^{1-F}}{\left(1-m^{-1} E_{\mathrm{BS}}\right)^{F}}=-\operatorname{sgn}(m)\left(\frac{|m|}{\mu}\right)^{2 F-1} A .
$$

Regular solutions (4.8) and (4.9) yield the following contribution to zeta function density (4.5):

$$
\begin{aligned}
{\left[\zeta_{\mathbf{x}}(z \mid m)\right]_{\mathrm{REG}}=} & \frac{1}{4 \pi} \int_{0}^{\infty} d k k|\tilde{E}|^{-2 z} \sum_{\operatorname{sgn}(\tilde{E})}\left\{\sum_{l=1}^{\infty}\left[\left(1+m \tilde{E}^{-1}\right) J_{l-F}^{2}(k r)+\left(1-m \tilde{E}^{-1}\right) J_{l+1-F}^{2}(k r)\right]+\right. \\
& \left.+\sum_{l^{\prime}=1}^{\infty}\left[\left(1+m \tilde{E}^{-1}\right) J_{l^{\prime}+F}^{2}(k r)+\left(1-m \tilde{E}^{-1}\right) J_{l^{\prime}-1+F}(k r)\right]\right\} .
\end{aligned}
$$

Summing over the energy sign and over $l$ and $l^{\prime}$, we get the expression

$$
\left[\zeta_{\mathbf{x}}(z \mid m)\right]_{\mathrm{REG}}=\frac{1}{\pi} \int_{0}^{\infty} d k k|\tilde{E}|^{-2 z} \int_{0}^{k r} \frac{d y}{y}\left[F J_{F}^{2}(y)+(1-F) J_{1-F}^{2}(y)\right],
$$

which in the case of $\operatorname{Re} z>1$ is reduced to the form

$$
\left[\zeta_{\mathbf{x}}(z \mid m)\right]_{\mathrm{REG}}=\frac{1}{2 \pi(z-1)} \int_{0}^{\infty} \frac{d k}{k}|\tilde{E}|^{2-2 z}\left[F J_{F}^{2}(k r)+(1-F) J_{1-F}^{2}(k r)\right] .
$$

Irregular solution (4.10) yields the following contribution to Eq.(4.5):

$$
\begin{gathered}
{\left[\zeta_{\mathbf{x}}(z \mid m)\right]_{\mathrm{IRREG}}=\frac{1}{4 \pi} \int_{0}^{\infty} d k k|\tilde{E}|^{-1-2 z}\left\{A \mu^{1-2 F} k^{2 F}\left[\tilde{L}_{(+)}-\tilde{L}_{(-)}\right] J_{-F}^{2}(k r)+\right.} \\
+A \mu^{1-2 F} k^{-2(1-F)}\left[(m-|\tilde{E}|)^{2} \tilde{L}_{(+)}-(m+|\tilde{E}|)^{2} \tilde{L}_{(-)}\right] J_{1-F}^{2}(k r)+
\end{gathered}
$$

\footnotetext{
${ }^{2}$ In a $2+1-$, as well as in any odd-, dimensional space-time mass parameter $m$ in Eq.(4.6) can take both positive and negative values.
} 


$$
\begin{aligned}
& +2\left[(m+|\tilde{E}|) \tilde{L}_{(+)}-(m-|\tilde{E}|) \tilde{L}_{(-)}\right] J_{-F}(k r) J_{F}(k r)+2\left[(m-|\tilde{E}|) \tilde{L}_{(+)}-\right. \\
& \left.-(m+|\tilde{E}|) \tilde{L}_{(-)}\right] J_{1-F}(k r) J_{-1+F}(k r)+A^{-1} \mu^{2 F-1} k^{-2 F}\left[(m+|\tilde{E}|)^{2} \tilde{L}_{(+)}-\right. \\
& \left.\left.-(m-|\tilde{E}|)^{2} \tilde{L}_{(-)}\right] J_{F}^{2}(k r)+A^{-1} \mu^{2 F-1} k^{2(1-F)}\left[\tilde{L}_{(+)}-\tilde{L}_{(-)}\right] J_{-1+F}^{2}(k r)\right\},
\end{aligned}
$$

where summation over the energy sign has been performed and

$$
\tilde{L}_{( \pm)}=\left[A \mu^{1-2 F} k^{-2(1-F)}(-m \pm|\tilde{E}|)+2 \cos (F \pi)+A^{-1} \mu^{2 F-1} k^{-2 F}(m \pm|\tilde{E}|)\right]^{-1}
$$

The contribution of bound state solution (4.13) to Eq.(4.5) is the following:

$$
\left[\zeta_{\mathbf{x}}(z \mid m)\right]_{\mathrm{BS}}=\frac{\sin (F \pi)}{\pi^{2}} \frac{\kappa^{2}\left|E_{\mathrm{BS}}\right|^{-2 z}}{m+E_{\mathrm{BS}}(2 F-1)}\left[\left(m+E_{\mathrm{BS}}\right) K_{F}^{2}(\kappa r)+\left(m-E_{\mathrm{BS}}\right) K_{1-F}^{2}(\kappa r)\right] .
$$

We show in Appendix C that Eq.(4.17) in the case of $1<\operatorname{Re} z<2$ is transformed to the following expression

$$
\begin{gathered}
{\left[\zeta_{\mathbf{x}}(z \mid m)\right]_{\mathrm{REG}}=\frac{|m|^{2(1-z)}}{2 \pi(z-1)}+\frac{\sin (z \pi)}{\pi^{2}(z-1)} r^{2(z-1)} \times} \\
\times \int_{|m| r}^{\infty} \frac{d w}{w}\left(w^{2}-m^{2} r^{2}\right)^{1-z}\left[F I_{F}(w) K_{F}(w)+(1-F) I_{1-F}(w) K_{1-F}(w)\right],
\end{gathered}
$$

while Eq.(4.18) in the case of $\frac{1}{2}<\operatorname{Re} z<1$ is transformed to the following one

$$
\begin{gathered}
{\left[\zeta_{\mathbf{x}}(z \mid m)\right]_{\mathrm{IRREG}}=\frac{\sin (z \pi)}{\pi^{2}} r^{2(z-1)} \int_{|m| r}^{\infty} d w w\left(w^{2}-m^{2} r^{2}\right)^{-z}\left[I_{F}(w) K_{F}(w)+I_{1-F}(w) K_{1-F}(w)\right]+} \\
+\frac{2 \sin (F \pi)}{\pi^{3}} \sin (z \pi) r^{2(z-1)} \times \\
\times \int_{|m| r}^{\infty} d w w\left(w^{2}-m^{2} r^{2}\right)^{-z} \frac{A \mu^{1-2 F}\left(\frac{w}{r}\right)^{2 F} K_{F}^{2}(w)+A^{-1} \mu^{2 F-1}\left(\frac{w}{r}\right)^{2(1-F)} K_{1-F}^{2}(w)}{A \mu^{1-2 F}\left(\frac{w}{r}\right)^{2 F}+2 m+A^{-1} \mu^{2 F-1}\left(\frac{w}{r}\right)^{2(1-F)}}- \\
\quad-\frac{\sin (F \pi)}{\pi^{2}} \frac{\kappa^{2}\left|E_{\mathrm{BS}}\right|^{-2 z}}{m+E_{\mathrm{BS}}(2 F-1)}\left[\left(m+E_{\mathrm{BS}}\right) K_{F}^{2}(\kappa r)+\left(m-E_{\mathrm{BS}}\right) K_{1-F}^{2}(\kappa r)\right] ;
\end{gathered}
$$

here $I_{\rho}(w)$ is the modified Bessel function of order $\rho$. The integral in Eq.(4.21) can be analytically continued to domain $\frac{1}{2}<\operatorname{Re} z<2$. In the case of $\frac{1}{2}<\operatorname{Re} z<1$ this integral is decomposed into two terms:

$$
\begin{gathered}
{\left[\zeta_{\mathbf{x}}(z \mid m)\right]_{\mathrm{REG}}=\frac{|m|^{2(1-z)}}{2 \pi(z-1)}-} \\
-\frac{\sin (z \pi)}{\pi^{2}} r^{2(z-1)} \int_{|m| r}^{\infty} d w w\left(w^{2}-m^{2} r^{2}\right)^{-z}\left[I_{F}(w) K_{F}(w)+I_{1-F}(w) K_{1-F}(w)\right]+
\end{gathered}
$$




$$
+\frac{2 \sin (F \pi)}{\pi^{3}} \frac{\sin (z \pi)}{z-1} r^{2(z-1)} \int_{|m| r}^{\infty} d w\left(w^{2}-m^{2} r^{2}\right)^{1-z} K_{F}(w) K_{1-F}(w),
$$

the last of which can be analytically continued to domain $\operatorname{Re} z<2$. Note also that the second integral in Eq.(4.22) can be analytically continued to domain $\operatorname{Re} z<1$.

Summing Eqs.(4.20), (4.22) and (4.23), we get

$$
\begin{gathered}
\zeta_{\mathbf{x}}(z \mid m)=\frac{|m|^{2(1-z)}}{2 \pi(z-1)}+\frac{2 \sin (F \pi)}{\pi^{3}} \frac{\sin (z \pi)}{z-1} r^{2(z-1)} \int_{|m| r}^{\infty} d w\left(w^{2}-m^{2} r^{2}\right)^{1-z} K_{F}(w) K_{1-F}(w)+ \\
+\frac{2 \sin (F \pi)}{\pi^{3}} \sin (z \pi) r^{2(z-1)} \times \\
\times \int_{|m| r}^{\infty} d w w\left(w^{2}-m^{2} r^{2}\right)^{-z} \frac{A \mu^{1-2 F}\left(\frac{w}{r}\right)^{2 F} K_{F}^{2}(w)+A^{-1} \mu^{2 F-1}\left(\frac{w}{r}\right)^{2(1-F)} K_{1-F}^{2}(w)}{A \mu^{1-2 F}\left(\frac{w}{r}\right)^{2 F}+2 m+A^{-1} \mu^{2 F-1}\left(\frac{w}{r}\right)^{2(1-F)}}
\end{gathered}
$$

i.e., the terms which are defined only in domain $\frac{1}{2}<\operatorname{Re} z<1$ are cancelled.

Note that the first term in Eq.(4.24) is identified with the zeta function density in the noninteracting theory (i.e. in the absence of any boundary condition and any background field):

$$
\zeta_{\mathbf{x}}^{(0)}(z \mid m)=\frac{|m|^{2(1-z)}}{2 \pi(z-1)}
$$

In the noninteracting theory all vacuum values are simply omitted due to the prescription of normal ordering of the product of operators (see, for example, 28]). Therefore, one has to subtract $\zeta_{\mathbf{x}}^{(0)}$ from $\zeta_{\mathbf{x}}$ for the reasons of consistency. Doing this and removing the infrared regulator mass, we obtain the renormalized zeta function density

$$
\begin{gathered}
\zeta_{\mathbf{x}}^{\mathrm{ren}}(z) \equiv \lim _{m \rightarrow 0}\left[\zeta_{\mathbf{x}}(z \mid m)-\zeta_{\mathbf{x}}^{(0)}(z \mid m)\right]= \\
=\frac{\sin (F \pi)}{\pi^{3}} \sin (z \pi) r^{2(z-1)}\left\{\frac{\sqrt{\pi}}{4} \frac{\Gamma(1-z)}{\Gamma\left(\frac{3}{2}-z\right)}\left[1-2 \frac{F(1-F)}{1-z}\right] \Gamma(F-z) \Gamma(1-F-z)+\right. \\
\left.+\int_{0}^{\infty} d w w^{1-2 z}\left[K_{F}^{2}(w)-K_{1-F}^{2}(w)\right] \tanh \left[(2 F-1) \ln \left(\frac{w}{\mu r}\right)+\ln A\right]\right\} .
\end{gathered}
$$

An alternative way of regularization of the infrared divergence in Eq.(4.4) is implemented by means of

$$
\zeta_{\mathbf{x}}(z \mid M)=\operatorname{tr}<\mathbf{x}|| H^{2}+\left.M^{2}\right|^{-z} \mid \mathbf{x}>
$$

where $M$ is the parameter of the dimension of mass, and it is implied that the complete set of solutions to the massless Dirac equation, (2.3) and (2.5), is used. Regular solutions (2.17) and (2.18) yield the following contribution to Eq.(4.27):

$$
\left[\zeta_{\mathbf{x}}(z \mid M)\right]_{\mathrm{REG}}=\frac{1}{2 \pi} \int_{0}^{\infty} d k k\left(k^{2}+M^{2}\right)^{-z}\left\{\sum_{l=1}^{\infty}\left[J_{l-F}^{2}(k r)+J_{l+1-F}^{2}(k r)\right]+\right.
$$




$$
\left.+\sum_{l^{\prime}=1}^{\infty}\left[J_{l^{\prime}+F}^{2}(k r)+J_{l^{\prime}-1+F}^{2}(k r)\right]\right\}
$$

where the summation over the energy sign has been performed. Following literally the same line, as in the derivation of Eq.(4.23), we get

$$
\begin{gathered}
{\left[\zeta_{\mathbf{x}}(z \mid M)\right]_{\mathrm{REG}}=} \\
=\frac{|M|^{2(1-z)}}{2 \pi(z-1)}-\frac{\sin (z \pi)}{\pi^{2}} r^{2(z-1)} \int_{|M| r}^{\infty} d w w\left(w^{2}-M^{2} r^{2}\right)^{-z}\left[I_{F}(w) K_{F}(w)+I_{1-F}(w) K_{1-F}(w)\right]+ \\
+\frac{2 \sin (F \pi)}{\pi^{3}} \frac{\sin (z \pi)}{z-1} r^{2(z-1)} \int_{|M| r}^{\infty} d w\left(w^{2}-M^{2} r^{2}\right)^{1-z} K_{F}(w) K_{1-F}(w) .
\end{gathered}
$$

Irregular solution (2.19) yields the following contribution to Eq.(4.27):

$$
\begin{aligned}
{\left[\zeta_{\mathbf{x}}(z \mid M)\right]_{\mathrm{IRREG}}=} & \frac{1}{4 \pi} \int_{0}^{\infty} d k k\left(k^{2}+M^{2}\right)^{-z}\left\{A\left(\frac{k}{\mu}\right)^{2 F-1}\left[L_{(+)}-L_{(-)}\right]\left[J_{-F}^{2}(k r)+J_{1-F}^{2}(k r)\right]+\right. \\
& +2\left[L_{(+)}+L_{(-)}\right]\left[J_{-F}(k r) J_{F}(k r)-J_{1-F}(k r) J_{-1+F}(k r)\right]+ \\
& \left.+A^{-1}\left(\frac{k}{\mu}\right)^{1-2 F}\left[L_{(+)}-L_{(-)}\right]\left[J_{F}^{2}(k r)+J_{-1+F}^{2}(k r)\right]\right\}
\end{aligned}
$$

where the summation over the energy sign has been performed and $L_{( \pm)}$is given by Eq.(3.6). We show in Appendix D that Eq.(4.30) at $\frac{1}{2}<\operatorname{Re} z<1$ is transformed to the following expression

$$
\begin{gathered}
{\left[\zeta_{\mathbf{x}}(z \mid M)\right]_{\text {IRREG }}=\frac{\sin (z \pi)}{\pi^{2}} r^{2(z-1)} \int_{|M| r}^{\infty} d w w\left(w^{2}-M^{2} r^{2}\right)^{-z}\left[I_{F}(w) K_{F}(w)+I_{1-F}(w) K_{1-F}(w)\right]+} \\
+\frac{\sin (F \pi)}{\pi^{3}} \sin (z \pi) r^{2(z-1)} \int_{|M| r}^{\infty} d w w\left(w^{2}-M^{2} r^{2}\right)^{-z}\left\{K_{F}^{2}(w)+K_{1-F}^{2}(w)+\right. \\
\left.+\left[K_{F}^{2}(w)-K_{1-F}^{2}(w)\right] \tanh \left[(2 F-1) \ln \left(\frac{w}{\mu r}\right)+\ln A\right]\right\} .
\end{gathered}
$$

Summing Eqs.(4.29) and (4.31), we get the expression

$$
\begin{aligned}
\zeta_{\mathbf{x}}(z \mid M)= & \frac{|M|^{2(1-z)}}{2 \pi(z-1)}+\frac{2 \sin (F \pi)}{\pi^{3}} \frac{\sin (z \pi)}{z-1} r^{2(z-1)} \int_{|M| r}^{\infty} d w\left(w^{2}-M^{2} r^{2}\right)^{1-z} K_{F}(w) K_{1-F}(w)+ \\
& +\frac{\sin (F \pi)}{\pi^{3}} \sin (z \pi) r^{2(z-1)} \int_{|M| r}^{\infty} d w w\left(w^{2}-M^{2} r^{2}\right)^{-z}\left\{K_{F}^{2}(w)+K_{1-F}^{2}(w)+\right.
\end{aligned}
$$




$$
\left.+\left[K_{F}^{2}(w)-K_{1-F}^{2}(w)\right] \tanh \left[(2 F-1) \ln \left(\frac{w}{\mu r}\right)+\ln A\right]\right\},
$$

which is analytically continued to domain $\operatorname{Re} z<1$.

One can see that the first term in Eq.(4.32) corresponding to the zeta function density in the noninteracting theory coincides with Eq.(4.25) under evident substitution $m \rightarrow M$, whereas the last integrals in Eqs.(4.32) and (4.24) differ essentially. However, the renormalized zeta function density

$$
\zeta_{\mathbf{x}}^{\mathrm{ren}}(z) \equiv \lim _{M \rightarrow 0}\left[\zeta_{\mathbf{x}}(z \mid M)-\zeta_{\mathbf{x}}^{(0)}(z \mid M)\right]
$$

coincides with that given by Eq.(4.26).

Although the former way of regularization of the infrared divergence, resulting in Eq.(4.24), looks much more consistent, the latter one, resulting in Eq.(4.32), appears to be technically simpler. Since both of them lead to the same results upon the removal of infrared regulators, we shall adopt, just for brevity, the latter one in the rest of the present paper. The only exception will be in the next section where the use of fermion mass $m$ as an infrared regulator for the effective action in 2+1-dimensional space-time is generically inevitable.

\section{ENERGY DENSITY AND EFFECTIVE POTENTIAL}

Recalling the formal expressions for the vacuum energy and zeta function densities, Eqs.(4.3) and (4.4), one can easily deduce that the physical (renormalized) vacuum energy density is expressed through the renormalized zeta function density at $z=-\frac{1}{2}$ :

$$
\mathcal{E}_{\mathbf{x}}^{\mathrm{ren}}=-\frac{1}{2} \zeta_{\mathbf{x}}^{\mathrm{ren}}\left(-\frac{1}{2}\right)
$$

In the background of a singular magnetic vortex (1.1) - (1.2), using Eq.(4.26), we get the expression

$$
\begin{gathered}
\mathcal{E}_{\mathbf{x}}^{\mathrm{ren}}=\frac{\sin (F \pi)}{2 \pi r^{3}}\left\{\frac{\frac{1}{2}-F}{6 \cos (F \pi)}\left[\frac{3}{4}-F(1-F)\right]+\right. \\
\left.+\frac{1}{\pi^{2}} \int_{0}^{\infty} d w w^{2}\left[K_{F}^{2}(w)-K_{1-F}^{2}(w)\right] \tanh \left[(2 F-1) \ln \left(\frac{w}{\mu r}\right)+\ln A\right]\right\} .
\end{gathered}
$$

At noninteger values of reduced vortex flux $\Phi^{(0)}-\Upsilon$ (i.e. at $0<F<1$ ) vacuum energy density (5.2) is positive. At half-integer values of the reduced vortex flux $\left(F=\frac{1}{2}\right)$ we get

$$
\left.\mathcal{E}_{\mathbf{x}}^{\text {ren }}\right|_{F=\frac{1}{2}}=\frac{1}{24 \pi^{2} r^{3}} \text {. }
$$

In the case of $\cos \Theta=0$ we get

$$
\mathcal{E}_{\mathbf{x}}^{\text {ren }}=\frac{\tan (F \pi)}{4 \pi r^{3}}\left(F-\frac{1}{2}\right)\left[\frac{1}{3} F(1-F)-\frac{1}{4} \mp \frac{1}{2}\left(F-\frac{1}{2}\right)\right], \quad \Theta= \pm s \frac{\pi}{2} .
$$

If $\cos \Theta \neq 0$, then at large distances from the vortex we get

$$
\mathcal{E}_{\mathbf{x}}^{\text {ren }} \underset{r \rightarrow \infty}{=} \frac{\tan (F \pi)}{4 \pi r^{3}}\left(F-\frac{1}{2}\right)\left[\frac{1}{3} F(1-F)-\frac{1}{4}+\frac{1}{2}\left|F-\frac{1}{2}\right|\right] .
$$

Going over to imaginary time $t=-i \tau$, let us consider the effective action in 2+1-dimensional Euclidean space-time:

$$
S_{(2+1)}^{\mathrm{eff}}[\mathbf{V}(\mathbf{x})]=-\ln \left\{N^{-1} \int d \Psi d \Psi^{+} \exp \left[-\int d \tau d^{2} x \Psi^{+}\left(-i \beta \partial_{\tau}-i \beta \tilde{H}\right) \Psi\right]\right\}=
$$




$$
=-\ln \operatorname{Det}\left[\left(-i \beta \partial_{\tau}-i \beta \tilde{H}\right) \tilde{m}^{-1}\right]
$$

here $N$ is a normalization factor, parameter $\tilde{m}$ is inserted just for the dimension reasons, while fermion mass $m$ (see Eq.(4.6)) is introduced in order to tame the infrared divergence. The real part of the effective action is presented in the form $\beta$

$$
\operatorname{Re} S_{(2+1)}^{\mathrm{eff}}[\mathbf{V}(\mathbf{x})]=-\frac{1}{2} \int d \tau d^{2} x \operatorname{tr}<\mathbf{x}, \tau\left|\ln \left[\left(-\partial_{\tau}^{2}+\tilde{H}^{2}\right) \tilde{m}^{-2}\right]\right| \mathbf{x}, \tau>.
$$

Let us define the zeta function density in threedimensional space $\left(x^{1}, x^{2}, \tau\right)$ :

$$
\zeta_{\mathbf{x}, \tau}(z \mid m)=\operatorname{tr}<\mathbf{x}, \tau\left|\left(-\partial_{\tau}^{2}+\tilde{H}^{2}\right)^{-z}\right| \mathbf{x}, \tau>.
$$

Then an ultraviolet regularization of Eq.(5.7) can be achieved by expressing its integrand through Eq.(5.8):

$$
-\frac{1}{2} \operatorname{tr}<\mathbf{x}, \tau\left|\ln \left[\left(-\partial_{\tau}^{2}+\tilde{H}^{2}\right) \tilde{m}^{-2}\right]\right| \mathbf{x}, \tau>=\left.\frac{1}{2}\left[\frac{d}{d z} \zeta_{\mathbf{x}, \tau}(z \mid m)\right]\right|_{z=0}+\frac{1}{2} \zeta_{\mathbf{x}, \tau}(0 \mid m) \ln \tilde{m}^{2} .
$$

In the background of a singular magnetic vortex (1.1) - (1.2) we get, similarly to Eq.(4.24), the following expression

$$
\begin{gathered}
\zeta_{\mathbf{x}, \tau}(z \mid m)=\frac{|m|^{3-2 z}}{4 \pi^{\frac{3}{2}}} \frac{\Gamma\left(z-\frac{3}{2}\right)}{\Gamma(z)}- \\
-\frac{\sin (F \pi)}{\pi^{\frac{7}{2}}} \cos (z \pi) \frac{\Gamma\left(z-\frac{3}{2}\right)}{\Gamma(z)} r^{2 z-3} \int_{|m| r}^{\infty} d w\left(w^{2}-m^{2} r^{2}\right)^{\frac{3}{2}-z} K_{F}(w) K_{1-F}(w)- \\
-\frac{\sin (F \pi)}{\pi^{\frac{7}{2}}} \cos (z \pi) \frac{\Gamma\left(z-\frac{1}{2}\right)}{\Gamma(z)} r^{2 z-3} \times \\
\times \int_{|m| r}^{\infty} d w w\left(w^{2}-m^{2} r^{2}\right)^{\frac{1}{2}-z} \frac{A \mu^{1-2 F}\left(\frac{w}{r}\right)^{2 F} K_{F}^{2}(w)+A^{-1} \mu^{2 F-1}\left(\frac{w}{r}\right)^{2(1-F)} K_{1-F}^{2}(w)}{A \mu^{1-2 F}\left(\frac{w}{r}\right)^{2 F}+2 m+A^{-1} \mu^{2 F-1}\left(\frac{w}{r}\right)^{2(1-F)}} ;
\end{gathered}
$$

note that the first term in Eq.(5.10) corresponds to the case of the noninteracting theory:

$$
\zeta_{\mathbf{x}, \tau}^{(0)}(z \mid m)=\frac{|m|^{3-2 z}}{4 \pi^{\frac{3}{2}}} \frac{\Gamma\left(z-\frac{3}{2}\right)}{\Gamma(z)} .
$$

Note also relation

$$
\zeta_{\mathbf{x}, \tau}(0 \mid m)=0,
$$

which ensures the independence of the effective action on $\tilde{m}^{2}$; thus Eq.(5.9) takes the form

$$
\begin{aligned}
& -\frac{1}{2} \operatorname{tr}\left\langle\mathbf{x}, \tau\left|\ln \left(-\partial_{\tau}^{2}+\tilde{H}^{2}\right) \tilde{m}^{-2}\right| \mathbf{x}, \tau\right\rangle=\frac{|m|^{3}}{6 \pi}-\frac{2 \sin (F \pi)}{3 \pi^{3} r^{3}} \int_{|m| r}^{\infty} d w\left(w^{2}-m^{2} r^{2}\right)^{\frac{3}{2}} K_{F}(w) K_{1-F}(w)+ \\
& \quad+\frac{\sin (F \pi)}{\pi^{3} r^{3}} \int_{|m| r}^{\infty} d w w\left(w^{2}-m^{2} r^{2}\right)^{\frac{1}{2}} \frac{A \mu^{1-2 F}\left(\frac{w}{r}\right)^{2 F} K_{F}^{2}(w)+A^{-1} \mu^{2 F-1}\left(\frac{w}{r}\right)^{2(1-F)} K_{1-F}^{2}(w)}{A \mu^{1-2 F}\left(\frac{w}{r}\right)^{2 F}+2 m+A^{-1} \mu^{2 F-1}\left(\frac{w}{r}\right)^{2(1-F)}} .
\end{aligned}
$$

\footnotetext{
${ }^{3}$ The imaginary part of the effective action vanishes in the case of a static background.
} 
The effective potential in the massless theory is defined as

$$
\mathcal{U}^{\mathrm{eff}}(\mathbf{x}, \tau)=-\frac{1}{2} \lim _{m \rightarrow 0} \operatorname{tr}\left\langle\mathbf{x}, \tau\left|\ln \left[\frac{\left(-\partial_{\tau}^{2}+\tilde{H}^{2}\right) \tilde{m}^{-2}}{\left(-\partial_{\tau}^{2}-\partial^{2}+m^{2}\right) \tilde{m}^{-2}}\right]\right| \mathbf{x}, \tau\right\rangle .
$$

Defining the renormalized zeta function density

$$
\zeta_{\mathbf{x}, \tau}^{\mathrm{ren}}(z)=\lim _{m \rightarrow 0}\left[\zeta_{\mathbf{x}, \tau}(z \mid m)-\zeta_{\mathbf{x}, \tau}^{(0)}(z \mid m)\right]
$$

we get, similarly to Eq.(5.9),

$$
\mathcal{U}^{\mathrm{eff}}(\mathbf{x}, \tau)=\left.\frac{1}{2}\left[\frac{d}{d z} \zeta_{\mathbf{x}, \tau}^{\mathrm{ren}}(z)\right]\right|_{z=0}+\frac{1}{2} \zeta_{\mathbf{x}, \tau}^{\mathrm{ren}}(0) \ln \tilde{m}^{2}
$$

In the background of a singular magnetic vortex (1.1) - (1.2), using Eq.(5.10), we get

$$
\begin{gathered}
\zeta_{\mathbf{x}, \tau}^{\mathrm{ren}}(z)=-\frac{\sin (F \pi)}{2 \pi^{\frac{7}{2}}} \cos (z \pi) \frac{\Gamma\left(z-\frac{1}{2}\right)}{\Gamma(z)} r^{2 z-3} \times \\
\times\left\{\frac{\sqrt{\pi}}{4} \frac{\Gamma\left(\frac{3}{2}-z\right)}{\Gamma(2-z)}\left[1-\frac{4 F(1-F)}{3-2 z}\right] \Gamma\left(\frac{1}{2}-z+F\right) \Gamma\left(\frac{3}{2}-z-F\right)+\right. \\
\left.+\int_{0}^{\infty} d w w^{2(1-z)}\left[K_{F}^{2}(w)-K_{1-F}^{2}(w)\right] \tanh \left[(2 F-1) \ln \left(\frac{w}{\mu r}\right)+\ln A\right]\right\}
\end{gathered}
$$

in particular

$$
\zeta_{\mathbf{x}, \tau}^{\mathrm{ren}}(0)=0
$$

and, thence, we arrive at the remarkable relation

$$
\mathcal{U}^{\mathrm{eff}}(\mathbf{x}, \tau)=\mathcal{E}_{\mathbf{x}}^{\mathrm{ren}}
$$

where $\mathcal{E}_{\mathbf{x}}^{\text {ren }}$ is given by Eq.(5.2).

Although the last relation looks rather natural and even evident, let us emphasize here that it is a consequence of the relation between the renormalized zeta function densities of different spatial dimensions,

$$
\left.\left[\frac{d}{d z} \zeta_{\mathbf{x}, \tau}^{\mathrm{ren}}(z)\right]\right|_{z=0}=-\zeta_{\mathbf{x}}^{\mathrm{ren}}\left(-\frac{1}{2}\right)
$$

and relation (5.18). As it has been shown in Ref. [29], relation (5.20) can be in general broken in spaces of higher dimensions. Moreover, both left- and right-hand sides of Eq.(5.20) can have nothing to do with the true vacuum energy density. Fortunately, this is not relevant for the case considered in the present paper, and, indeed, in the background of a singular magnetic vortex the vacuum energy density coincides with the effective potential.

\section{CURRENT}

Let us regard the 2-dimensional space $\left(x^{1}, x^{2}\right)$ as a $1+1$-dimensional space-time with the Wick-rotated time axis. The Clifford algebra in this space-time has the exact irreducible representation with the above $\alpha$ matrices playing now the role of the $\gamma$ matrices and the $\beta$ matrix playing the role similar to that of the $\gamma^{5}$ matrix in a $3+1$-dimensional 
space-time. Introducing mass parameter $M$ to tame the infrared divergence, one can define the trace of two-point causal Green's function with the $\alpha$ matrix inserted between the field operators:

$$
\mathbf{J}\left(\mathbf{x}, \mathbf{x}^{\prime} \mid M\right)=<\operatorname{vac}\left|T \Psi^{+}\left(\mathbf{x}^{\prime}, 0\right) \boldsymbol{\alpha} \Psi(\mathbf{x}, 0)\right| \operatorname{vac}>=\operatorname{tr}<\mathbf{x}\left|\boldsymbol{\alpha}(H-i M)^{-1}\right| \mathbf{x}^{\prime}>,
$$

where $T$ is the symbol of time ordering in the 1+1-dimensional space-time. Inserting damping factor $\left(E^{2}+M^{2}\right)^{-z}$ (where $\operatorname{Re} z>0$ ) into the integral corresponding to Eq.(6.1), we define matrix element

$$
\mathbf{J}\left(\mathbf{x}, \mathbf{x}^{\prime} ; z \mid M\right)=\operatorname{tr}<\mathbf{x}\left|\boldsymbol{\alpha}(H+i M)\left(H^{2}+M^{2}\right)^{-1-z}\right| \mathbf{x}^{\prime}>.
$$

Returning to the massless theory in the 2+1-dimensional space-time $\left(x^{1}, x^{2}, t\right)$, let us define the vacuum current in the conventional way (compare with Eqs. (3.1) - (3.3))

$$
\mathbf{j}(\mathbf{x})=<\operatorname{vac}\left|\frac{1}{2}\left[\Psi^{+}(\mathbf{x}, t), \boldsymbol{\alpha} \Psi(\mathbf{x}, t)\right]_{-}\right| \operatorname{vac}>=-\frac{1}{2} \operatorname{tr}<\mathbf{x}|\boldsymbol{\alpha} \operatorname{sgn}(H)| \mathbf{x}>.
$$

One can notice relation

$$
\mathbf{j}(\mathbf{x})=-\frac{1}{2} \mathbf{J}\left(\mathbf{x}, \mathbf{x} ;-\frac{1}{2} \mid 0\right)
$$

so that in the following the matrix element (6.2) in the coincidence limit $\mathbf{x}^{\prime}=\mathbf{x}$ will be regarded as a generalized current.

In the background of a singular magnetic vortex (1.1) - (1.2) radial component

$$
J_{r}(\mathbf{x}, \mathbf{x} ; z \mid M)=r^{-1}\left[x^{1} J_{1}(\mathbf{x}, \mathbf{x} ; z \mid M)+x^{2} J_{2}(\mathbf{x}, \mathbf{x} ; z \mid M)\right]
$$

vanishes, whereas angular component

$$
J_{\varphi}(\mathbf{x}, \mathbf{x} ; z \mid M)=r^{-1}\left[x^{1} J_{2}(\mathbf{x}, \mathbf{x} ; z \mid M)-x^{2} J_{1}(\mathbf{x}, \mathbf{x} ; z \mid M)\right]
$$

is nonvanishing. The contribution of regular solutions (2.17) and (2.18) to Eq.(6.6) is given by the expression

$$
\begin{aligned}
{\left[J_{\varphi}(\mathbf{x}, \mathbf{x} ; z \mid M)\right]_{\mathrm{REG}}=} & \frac{s}{\pi} \int_{0}^{\infty} d k \frac{k^{2}}{\left(k^{2}+M^{2}\right)^{1+z}}\left[\sum_{l=1}^{\infty} J_{l-F}(k r) J_{l+1-F}(k r)-\right. \\
& \left.-\sum_{l^{\prime}=1}^{\infty} J_{l^{\prime}+F}(k r) J_{l^{\prime}-1+F}(k r)\right] .
\end{aligned}
$$

Performing the summation over $l$ and $l^{\prime}$, we get

$$
\begin{gathered}
{\left[J_{\varphi}(\mathbf{x}, \mathbf{x} ; z \mid M)\right]_{\mathrm{REG}}=\frac{s}{\pi} \int_{0}^{\infty} d k k^{2}\left(k^{2}+M^{2}\right)^{-1-z}\left\{F J_{F}(k r) J_{-1+F}(k r)-\right.} \\
\left.-(1-F) J_{1-F}(k r) J_{-F}(k r)-\frac{1}{2} k r\left[J_{F}^{2}(k r)+J_{-1+F}^{2}(k r)-J_{-F}^{2}(k r)-J_{1-F}^{2}(k r)\right]\right\} .
\end{gathered}
$$

Transforming the integral in the last expression similarly to that as in derivation of $\left[\zeta_{\mathbf{x}}(z \mid M)\right]_{\mathrm{REG}}(4.29)$ in Section IV, we get

$$
\left[J_{\varphi}(\mathbf{x}, \mathbf{x} ; z \mid M)\right]_{\mathrm{REG}}=\frac{s \sin (z \pi)}{\pi^{2}} r^{2 z-1} \int_{|M| r}^{\infty} d w w^{2}\left(w^{2}-M^{2} r^{2}\right)^{-1-z} \times
$$




$$
\begin{gathered}
\times\left\{I_{F}(w) K_{1-F}(w)-I_{1-F}(w) K_{F}(w)+\right. \\
\left.+\frac{2 \sin (F \pi)}{\pi}\left[w K_{F}^{2}(w)-w K_{1-F}^{2}(w)-(2 F-1) K_{F}(w) K_{1-F}(w)\right]\right\} .
\end{gathered}
$$

The contribution of irregular solution (2.19) to Eq.(6.6) is given by expression

$$
\begin{gathered}
{\left[J_{\varphi}(\mathbf{x}, \mathbf{x} ; z \mid M)\right]_{\text {IRREG }}=\frac{s}{2 \pi} \int_{0}^{\infty} d k k\left(k^{2}+M^{2}\right)^{-1-z} \times} \\
\times\left\{A\left(\frac{k}{\mu}\right)^{2 F-1}\left[(k+i M) L_{(+)}-(k-i M) L_{(-)}\right] J_{-F}(k r) J_{1-F}(k r)+\right. \\
+\left[(k+i M) L_{(+)}+(k-i M) L_{(-)}\right]\left[J_{F}(k r) J_{1-F}(k r)-J_{-F}(k r) J_{-1+F}(k r)\right]- \\
\left.-A^{-1}\left(\frac{k}{\mu}\right)^{1-2 F}\left[(k+i M) L_{(+)}-(k-i M) L_{(-)}\right] J_{F}(k r) J_{-1+F}(k r)\right\},
\end{gathered}
$$

where $A$ and $L_{( \pm)}$are given by Eqs.(3.5) and (3.6), respectively. Transforming the integral in Eq.(6.10) similarly to that as in Appendix D, we get

$$
\begin{aligned}
& {\left[J_{\varphi}(\mathbf{x}, \mathbf{x} ; z \mid M)\right]_{\text {IRREG }}=\frac{s \sin (z \pi)}{\pi^{2}} r^{2 z-1} \int_{|M| r}^{\infty} d w w^{2}\left(w^{2}-M^{2} r^{2}\right)^{-1-z} \times} \\
& \times\left(I_{1-F}(w) K_{F}(w)-I_{F}(w) K_{1-F}(w)-\frac{2 \sin (F \pi)}{\pi} K_{F}(w) K_{1-F}(w) \times\right. \\
& \left.\times\left\{\tanh \left[(2 F-1) \ln \left(\frac{w}{\mu r}\right)+\ln A\right]-\frac{i M r}{w \cosh \left[(2 F-1) \ln \left(\frac{w}{\mu r}\right)+\ln A\right]}\right\}\right) .
\end{aligned}
$$

Summing Eqs.(6.9) and (6.11), we get

$$
\begin{gathered}
J_{\varphi}(\mathbf{x}, \mathbf{x} ; z \mid M)=\frac{2 s \sin (F \pi)}{\pi^{3}} \sin (z \pi) r^{2 z-1} \int_{|M| r}^{\infty} d w w^{2}\left(w^{2}-M^{2} r^{2}\right)^{-1-z} \times \\
\times\left(w\left[K_{F}^{2}(w)-K_{1-F}^{2}(w)\right]-K_{F}(w) K_{1-F}(w)\{2 F-1+\right. \\
\left.\left.+\tanh \left[(2 F-1) \ln \left(\frac{w}{\mu r}\right)+\ln A\right]-\frac{i M r}{w \cosh \left[(2 F-1) \ln \left(\frac{w}{\mu r}\right)+\ln A\right]}\right\}\right) .
\end{gathered}
$$

Note that Eqs.(6.9) and (6.11) are valid at $-\frac{1}{2}<\operatorname{Re} z<0$, and their sum, Eq.(6.12), can be analytically continued to half-plane $\operatorname{Re} z<0$. This may look somewhat embarrassing, since the initial motivation, as presented above, was to consider the case of $\operatorname{Re} z>0$. However, the situation can be cured by means of analytic continuation using partial integration. Namely, integrating Eq.(6.12) by parts, we get the expression

$$
J_{\varphi}(\mathbf{x}, \mathbf{x} ; z \mid M)=-\frac{s \sin (F \pi)}{\pi^{3}} \frac{\sin (z \pi)}{z} r^{2 z-1} \int_{|M| r}^{\infty} d w\left(w^{2}-M^{2} r^{2}\right)^{-z} \times
$$




$$
\begin{aligned}
& \times\left[w\left[K_{F}^{2}(w)-K_{1-F}^{2}(w)\right]+\left[K_{F}^{2}(w)+K_{1-F}^{2}(w)\right]\left\{w \tanh \left[(2 F-1) \ln \left(\frac{w}{\mu r}\right)+\ln A\right]-\right.\right. \\
& \left.-\frac{i M r}{\cosh \left[(2 F-1) \ln \left(\frac{w}{\mu r}\right)+\ln A\right]}\right\}-\frac{K_{F}(w) K_{1-F}(w)}{\cosh \left[(2 F-1) \ln \left(\frac{w}{\mu r}\right)+\ln A\right]} \times \\
& \left.\times\left(\frac{2 F-1}{\cosh \left[(2 F-1) \ln \left(\frac{w}{\mu r}\right)+\ln A\right]}+\frac{i M r}{w}\left\{(2 F-1) \tanh \left[(2 F-1) \ln \left(\frac{w}{\mu r}\right)+\ln A\right]+1\right\}\right)\right],
\end{aligned}
$$

which is analytically continued to domain $\operatorname{Re} z<1$. Integrating Eq.(6.12) by parts $N$ times, one can get the expression for the generalized current which is analytically continued to domain $\operatorname{Re} z<N$.

Taking into account Eq.(6.4), we get the following expression for the vacuum current:

$$
j_{\varphi}(\mathbf{x})=\frac{s \sin (F \pi)}{\pi r^{2}}\left\{\frac{\left(F-\frac{1}{2}\right)^{2}}{4 \cos (F \pi)}-\frac{1}{\pi^{2}} \int_{0}^{\infty} d w w K_{F}(w) K_{1-F}(w) \tanh \left[\left[(2 F-1) \ln \left(\frac{w}{\mu r}\right)+\ln A\right]\right\} ;\right.
$$

recall that radial component $j_{r}(\mathbf{x})$ is vanishing, see Eq.(6.5). At $\cos \Theta=0$ we get

$$
j_{\varphi}(\mathbf{x})=\frac{s \tan (F \pi)}{4 \pi r^{2}}\left(F-\frac{1}{2}\right)\left(F-\frac{1}{2} \pm 1\right), \quad \Theta= \pm s \frac{\pi}{2} .
$$

At half-integer values of the reduced vortex flux $\left(F=\frac{1}{2}\right)$, taking into account relation

$$
\left.A\right|_{F=\frac{1}{2}}=\tan \left(s \frac{\Theta}{2}+\frac{\pi}{4}\right)
$$

we get

$$
\left.j_{\varphi}(\mathbf{x})\right|_{F=\frac{1}{2}}=-\frac{\sin \Theta}{4 \pi^{2} r^{2}} .
$$

If $\cos \Theta \neq 0$ and $F \neq \frac{1}{2}$, then at large distances from the vortex we get

$$
j_{\varphi}(\mathbf{x})_{r \rightarrow \infty}=\frac{s \tan (F \pi)}{4 \pi r^{2}}\left|F-\frac{1}{2}\right|\left(\left|F-\frac{1}{2}\right|-1\right) .
$$

\section{PARITY BREAKING CONDENSATE}

Since twodimensional massless Dirac Hamiltonian (2.5) anticommutes with the $\beta$ matrix

$$
[H, \beta]_{+}=0,
$$

the Dirac equation (2.3) is invariant under the parity transformation

$$
E_{\lambda} \rightarrow-E_{\lambda}, \quad<\mathbf{x}|\lambda>\rightarrow \beta<\mathbf{x}| \lambda>.
$$

However, this invariance is violated by boundary condition (2.14), unless $\cos \Theta=0$. Consequently, the parity breaking condensate emerges in the vacuum:

$$
\mathcal{C}_{\mathbf{x}}=<\operatorname{vac}\left|\frac{1}{2}\left[\Psi^{+}(\mathbf{x}, t), \beta \Psi(\mathbf{x}, t)\right]_{-}\right| \operatorname{vac}>=-\frac{1}{2} \operatorname{tr}<\mathbf{x}|\beta \operatorname{sgn}(H)| \mathbf{x}>.
$$

Let us start with the regularized condensate

$$
\mathcal{C}_{\mathbf{x}}(z \mid M)=-\frac{1}{2} \operatorname{tr}<\mathbf{x}\left|\beta H\left(H^{2}+M^{2}\right)^{-\frac{1}{2}-z}\right| \mathbf{x}>.
$$


The contribution of regular solutions (2.17) and (2.18) to Eq.(7.4) is cancelled upon summation over the sign of energy. Thus, only the contribution of irregular solution (2.19) to Eq.(7.4) survives:

$$
\begin{aligned}
\mathcal{C}_{\mathbf{x}}(z \mid M)=-\frac{1}{8 \pi} & \int_{0}^{\infty} d k k^{2}\left(k^{2}+M^{2}\right)^{-\frac{1}{2}-z}\left\{A\left(\frac{k}{\mu}\right)^{2 F-1}\left[L_{(+)}+L_{(-)}\right]\left[J_{-F}^{2}(k r)-J_{1-F}^{2}(k r)\right]+\right. \\
+ & 2\left[L_{(+)}-L_{(-)}\right]\left[J_{-F}(k r) J_{F}(k r)+J_{1-F}(k r) J_{-1+F}(k r)\right]+ \\
& \left.+A^{-1}\left(\frac{k}{\mu}\right)^{1-2 F}\left[L_{(+)}+L_{(-)}\right]\left[J_{F}^{2}(k r)-J_{-1+F}^{2}(k r)\right]\right\},
\end{aligned}
$$

where $A$ and $L_{( \pm)}$are given by Eqs.(3.5) and (3.6), respectively. Transforming the integral in Eq.(7.5) similarly to that as in Appendix D, we get

$$
\mathcal{C}_{\mathbf{x}}(z \mid M)=-\frac{\sin (F \pi)}{2 \pi^{3}} \cos (z \pi) r^{2(z-1)} \int_{|M| r}^{\infty} d w w^{2}\left(w^{2}-M^{2} r^{2}\right)^{-\frac{1}{2}-z} \frac{K_{F}^{2}(w)+K_{1-F}^{2}(w)}{\cosh \left[(2 F-1) \ln \left(\frac{w}{\mu r}\right)+\ln A\right]},
$$

where $\operatorname{Re} z<\frac{1}{2}$. Then vacuum condensate (7.3) is given by the following expression:

$$
\mathcal{C}_{\mathbf{x}} \equiv \mathcal{C}_{\mathbf{x}}(0 \mid 0)=-\frac{\sin (F \pi)}{2 \pi^{3} r^{2}} \int_{0}^{\infty} d w w \frac{K_{F}^{2}(w)+K_{1-F}^{2}(w)}{\cosh \left[(2 F-1) \ln \left(\frac{w}{\mu r}\right)+\ln A\right]}
$$

Evidently, Eq.(7.7) vanishes at $\cos \Theta=0$. At half-integer values of the reduced vortex flux $\left(F=\frac{1}{2}\right)$ we get

$$
\left.\mathcal{C}_{\mathbf{x}}\right|_{F=\frac{1}{2}}=-\frac{\cos \Theta}{4 \pi^{2} r^{2}}
$$

If $\cos \Theta \neq 0$ and $F \neq \frac{1}{2}$, then at large distances from the vortex we get

$$
\mathcal{C}_{\mathbf{x}_{r \rightarrow \infty} \equiv}-\frac{\sin (F \pi)}{2 \pi^{2} r^{2}}\left\{\begin{array}{c}
(\mu r)^{2 F-1} A^{-1} \frac{\Gamma\left(\frac{3}{2}-F\right) \Gamma\left(\frac{3}{2}-2 F\right)}{\Gamma(1-F)}, 0<F<\frac{1}{2} \\
(\mu r)^{1-2 F} A \frac{\Gamma\left(F+\frac{1}{2}\right) \Gamma\left(2 F-\frac{1}{2}\right)}{\Gamma(F)}, \quad \frac{1}{2}<F<1
\end{array} .\right.
$$

Integrating Eq.(7.7) over the plane $\left(x^{1}, x^{2}\right)$, we obtain the total vacuum condensate

$$
\mathcal{C} \equiv \int d^{2} x \mathcal{C}_{\mathbf{x}}=-\frac{\operatorname{sgn}_{0}(\cos \Theta)}{4\left|F-\frac{1}{2}\right|} .
$$

Thus, the total vacuum condensate is infinite at $F=\frac{1}{2}$ if $\cos \Theta \neq 0$.

\section{ABSENCE OF PARITY ANOMALY}

Let us define the generalized twodimensional axial current

$$
\mathbf{J}^{3}(\mathbf{x}, \mathbf{x} ; z \mid M)=i \operatorname{tr}<\mathbf{x}\left|\boldsymbol{\alpha} \beta(H+i M)\left(H^{2}+M^{2}\right)^{-1-z}\right| \mathbf{x}>.
$$

Owing to the twodimensionality, the components of current (8.1) are related to the components of the generalized current considered in Section VI (Eq.(6.2) at $\mathbf{x}^{\prime}=\mathbf{x}$ )

$$
J_{r}^{3}=s J_{\varphi}, \quad J_{\varphi}^{3}=-s J_{r}
$$


In the background of a singular magnetic vortex (1.1) - (1.2) the divergence of the current $\mathbf{J}$ is vanishing (see Eq.(6.12)):

$$
\partial \cdot \mathbf{J}(\mathbf{x}, \mathbf{x} ; z \mid M)=0
$$

whereas the divergence of the current $\mathbf{J}^{3}$ is nonvanishing, owing to the relation

$$
\boldsymbol{\partial} \cdot \mathbf{J}^{3}(\mathbf{x}, \mathbf{x} ; z \mid M)=s \boldsymbol{\partial} \times \mathbf{J}(\mathbf{x}, \mathbf{x} ; z \mid M) .
$$

Let us consider the effective action in 1+1-dimensional Euclidean space-time

$$
S_{(1+1)}^{\mathrm{eff}}[\mathbf{V}(\mathbf{x})]=-\int d^{2} x \operatorname{tr}<\mathbf{x}\left|\ln \left(H \tilde{M}^{-1}\right)\right| \mathbf{x}>,
$$

where $\tilde{M}$ is the parameter of the dimension of mass. The invariance of action (8.5) under the gauge transformation,

$$
\begin{gathered}
V(\mathbf{x}) \rightarrow \mathbf{V}(\mathbf{x})+\boldsymbol{\partial} \Lambda(\mathbf{x}), \\
<\mathbf{x}\left|\rightarrow e^{i \Lambda(\mathbf{x})}<\mathbf{x}\right|, \quad\left|\mathbf{x}>\rightarrow e^{-i \Lambda(\mathbf{x})}\right| \mathbf{x}>,
\end{gathered}
$$

is stipulated by conservation law

$$
\lim _{\substack{M \rightarrow 0 \\ z \rightarrow 0}} \partial \cdot \mathbf{J}(\mathbf{x}, \mathbf{x} ; z \mid M)=0
$$

If conservation law

$$
\lim _{\substack{M \rightarrow 0 \\ z \rightarrow 0}} \partial \cdot \mathbf{J}^{3}(\mathbf{x}, \mathbf{x} ; z \mid M)=0
$$

holds, then action (8.5) is invariant under the localized version of the parity transformation (compare with Eq.(7.2))

$$
\begin{gathered}
\mathbf{V}(\mathbf{x}) \rightarrow \mathbf{V}(\mathbf{x})+\boldsymbol{\partial} \beta \Lambda(\mathbf{x}) \\
<\mathbf{x}\left|\rightarrow e^{i \beta \Lambda(\mathbf{x})}<\mathbf{x}\right|, \quad|\mathbf{x}>\rightarrow| \mathbf{x}>e^{i \beta \Lambda(\mathbf{x})} .
\end{gathered}
$$

The breakdown of the latter symmetry,

$$
\lim _{\substack{M \rightarrow 0 \\ z \rightarrow 0}} \boldsymbol{\partial} \cdot \mathbf{J}^{3}(\mathbf{x}, \mathbf{x} ; z \mid M) \neq 0
$$

is denoted as a parity anomaly, i.e., the axial anomaly in 1+1-dimensional space-time.

Note that in classical theory both gauge and localized parity symmetries are conserved. This is reflected by the formal invariance of action (8.5) under both transformations (8.6) and (8.9). However, in quantum theory, in order to calculate the effective action in a certain background, one has to use regularization which in fact breaks the localized parity symmetry. Namely, one has to substitute $\ln \left[(H-i M) \tilde{M}^{-1}\right]$ for $\ln \left(H \tilde{M}^{-1}\right)$ into Eq.(8.5) (compare with Eqs.(5.6)-(5.7)), where regulator mass $M$ is the symmetry breaking parameter. That is why generalized currents (6.2) at $\mathbf{x}^{\prime}=\mathbf{x}$ and (8.1) come into play. The divergence of the latter current can be presented in the form

$$
\partial \cdot \mathbf{J}^{3}(\mathbf{x}, \mathbf{x} ; z \mid M)=2 \tilde{\zeta}_{\mathbf{x}}(z \mid M)-2 M^{2} \tilde{\zeta}_{\mathbf{x}}(z+1 \mid M)-4 i M \mathcal{C}_{\mathbf{x}}\left(z+\frac{1}{2} \mid M\right),
$$

where $\mathcal{C}_{\mathbf{x}}(z \mid M)$ is regularized condensate (7.4) and

$$
\tilde{\zeta}_{\mathbf{x}}(z \mid M)=\operatorname{tr}<\mathbf{x}\left|\beta\left(H^{2}+M^{2}\right)^{-z}\right| \mathbf{x}>
$$


is the modified (by insertion of the $\beta$ matrix) zeta function density.

In the background of a singular magnetic vortex, conservation law (8.7) holds, as a consequence of Eq.(8.3). Incidentally, the regularized condensate is given by Eq.(7.6). As to the modified zeta function density, we get the following expression for the contribution of regular solutions (2.17) and (2.18) to Eq.(8.12):

$$
\begin{aligned}
{\left[\tilde{\zeta}_{\mathbf{x}}(z \mid M)\right]_{\mathrm{REG}}=\frac{1}{2 \pi} } & \int_{0}^{\infty} d k k\left(k^{2}+M^{2}\right)^{-z}\left\{\sum_{l=1}^{\infty}\left[J_{l-F}^{2}(k r)-J_{l+1-F}^{2}(k r)\right]+\right. \\
& \left.+\sum_{l^{\prime}=1}^{\infty}\left[J_{l^{\prime}+F}^{2}(k r)-J_{l^{\prime}-1+F}^{2}(k r)\right]\right\},
\end{aligned}
$$

which, upon summation over $l$ and $l^{\prime}$, takes the form

$$
\left[\tilde{\zeta}_{\mathbf{x}}(z \mid M)\right]_{\mathrm{REG}}=\frac{1}{2 \pi} \int_{0}^{\infty} d k k\left(k^{2}+M^{2}\right)^{-z}\left[J_{1-F}^{2}(k r)-J_{F}^{2}(k r)\right] .
$$

Irregular solution (2.19) yields the following contribution to Eq.(8.12):

$$
\begin{gathered}
{\left[\tilde{\zeta}_{\mathbf{x}}(z \mid M)\right]_{\text {IRREG }}=\frac{1}{4 \pi} \int_{0}^{\infty} d k k\left(k^{2}+M^{2}\right)^{-z}\left\{A\left(\frac{k}{\mu}\right)^{2 F-1}\left[L_{(+)}-L_{(-)}\right] \times\right.} \\
\times\left[J_{-F}^{2}(k r)-J_{1-F}^{2}(k r)\right]+2\left[L_{(+)}+L_{(-)}\right]\left[J_{-F}(k r) J_{F}(k r)+J_{1-F}(k r) J_{-1+F}(k r)\right]+ \\
\left.+A^{-1}\left(\frac{k}{\mu}\right)^{1-2 F}\left[L_{(+)}-L_{(-)}\right]\left[J_{F}^{2}(k r)-J_{-1+F}^{2}(k r)\right]\right\},
\end{gathered}
$$

where $A$ and $L_{( \pm)}$are given by Eqs.(3.5) and (3.6). Similarly to the case of zeta function density $\zeta_{\mathbf{x}}(z \mid M)(4.27)$ (see Section IV and Appendix D), we recast Eqs.(8.14) and (8.15) for $\frac{1}{2}<\operatorname{Re} z<1$ into the following form

$$
\left[\tilde{\zeta}_{\mathbf{x}}(z \mid M)\right]_{\mathrm{REG}}=\frac{\sin (z \pi)}{\pi^{2}} r^{2(z-1)} \int_{|M| r}^{\infty} d w w\left(w^{2}-M^{2} r^{2}\right)^{-z}\left[I_{1-F}(w) K_{1-F}(w)-I_{F}(w) K_{F}(w)\right]
$$

and

$$
\begin{gathered}
{\left[\tilde{\zeta}_{\mathbf{x}}(z \mid M)\right]_{\text {IRREG }}=\frac{\sin (z \pi)}{\pi^{2}} r^{2(z-1)} \int_{|M| r}^{\infty} d w w\left(w^{2}-M^{2} r^{2}\right)^{-z}\left[I_{F}(w) K_{F}(w)-I_{1-F}(w) K_{1-F}(w)\right]+} \\
+\frac{\sin (F \pi)}{\pi^{3}} \sin (z \pi) r^{2(z-1)} \int_{|M| r}^{\infty} d w w\left(w^{2}-M^{2} r^{2}\right)^{-z}\left\{K_{F}^{2}(w)-K_{1-F}^{2}(w)+\right. \\
\left.+\left[K_{F}^{2}(w)+K_{1-F}^{2}(w)\right] \tanh \left[(2 F-1) \ln \left(\frac{w}{\mu r}\right)+\ln A\right]\right\},
\end{gathered}
$$

resulting in the expression

$$
\tilde{\zeta}_{\mathbf{x}}(z \mid M)=\frac{\sin (F \pi)}{\pi^{3}} \sin (z \pi) r^{2(z-1)} \int_{|M| r}^{\infty} d w w\left(w^{2}-M^{2} r^{2}\right)^{-z} \times
$$




$$
\times\left\{K_{F}^{2}(w)-K_{1-F}^{2}(w)+\left[K_{F}^{2}(w)+K_{1-F}^{2}(w)\right] \tanh \left[(2 F-1) \ln \left(\frac{w}{\mu r}\right)+\ln A\right]\right\},
$$

which is analytically continued to domain $\operatorname{Re} z<1$.

Extending the domain of definition in $z$ in Eqs.(7.6) and (8.18) by means of integration by parts, we get

$$
\begin{aligned}
\mathcal{C}_{\mathbf{x}}(z \mid M) & =-\frac{\sin (F \pi)}{\pi^{3}} \frac{\cos (z \pi)}{1-2 z} r^{2(z-1)} \int_{|M| r}^{\infty} \frac{d w\left(w^{2}-M^{2} r^{2}\right)^{\frac{1}{2}-z}}{\cosh \left[(2 F-1) \ln \left(\frac{w}{\mu r}\right)+\ln A\right]} \times \\
& \times\left\{\left(F-\frac{1}{2}\right)\left[K_{F}^{2}(w)-K_{1-F}^{2}(w)\right]+2 w K_{F}(w) K_{1-F}(w)+\right. \\
+ & \left.\left(F-\frac{1}{2}\right)\left[K_{F}^{2}(w)+K_{1-F}^{2}(w)\right] \tanh \left[(2 F-1) \ln \left(\frac{w}{\mu r}\right)+\ln A\right]\right\},
\end{aligned}
$$

where $\operatorname{Re} z<\frac{3}{2}$ and

$$
\begin{gathered}
\tilde{\zeta}_{\mathbf{x}}(z \mid M)=\frac{\sin (F \pi)}{\pi^{3}} \frac{\sin (z \pi)}{1-z} r^{2(z-1)} \int_{|M| r}^{\infty} \frac{d w}{w}\left(w^{2}-M^{2} r^{2}\right)^{1-z} \times \\
\times\left\{\frac{1}{2}\left[K_{F}^{2}(w)-K_{1-F}^{2}(w)\right]+\left[F K_{F}^{2}(w)+(1-F) K_{1-F}^{2}+2 w K_{F}(w) K_{1-F}(w)\right] \times\right. \\
\left.\times \tanh \left[(2 F-1) \ln \left(\frac{w}{\mu r}\right)+\ln A\right]+\left(F-\frac{1}{2}\right)\left[K_{F}^{2}(w)+K_{1-F}^{2}(w)\right] \tanh ^{2}\left[(2 F-1) \ln \left(\frac{w}{\mu r}\right)+\ln A\right]\right\},
\end{gathered}
$$

where $\operatorname{Re} z<2$. Using the latter representations, we get

$$
\lim _{M \rightarrow 0} M \mathcal{C}_{\mathbf{x}}\left(z+\frac{1}{2} \mid M\right)=\lim _{M \rightarrow 0} M^{2} \tilde{\zeta}_{\mathbf{x}}(z+1 \mid M)=0, \quad \operatorname{Re} z<1,
$$

and, consequently,

$$
\lim _{M \rightarrow 0} \boldsymbol{\partial} \cdot \mathbf{J}^{3}(\mathbf{x}, \mathbf{x} ; z \mid M)=2 \tilde{\zeta}_{\mathbf{x}}(z \mid 0), \quad \operatorname{Re} z<1
$$

One can easily get

$$
\begin{aligned}
\tilde{\zeta}_{\mathbf{x}}(z \mid 0) & =\frac{\sin (F \pi)}{\pi^{3}} \sin (z \pi) r^{2(z-1)}\left\{\frac{\sqrt{\pi}}{2} \frac{\Gamma(1-z)}{\Gamma\left(\frac{3}{2}-z\right)}\left(F-\frac{1}{2}\right) \Gamma(F-z) \Gamma(1-F-z)+\right. \\
& \left.+\int_{0}^{\infty} d w w^{1-2 z}\left[K_{F}^{2}(w)+K_{1-F}^{2}(w)\right] \tanh \left[(2 F-1) \ln \left(\frac{w}{\mu r}\right)+\ln A\right]\right\} ;
\end{aligned}
$$

in particular, at half-integer values of the reduced vortex flux:

$$
\left.\tilde{\zeta}_{\mathbf{x}}(z \mid 0)\right|_{F=\frac{1}{2}}=\frac{s \sin \Theta}{2 \pi^{\frac{3}{2}}} \frac{\Gamma\left(\frac{1}{2}-z\right)}{\Gamma(z)} r^{2(z-1)} ;
$$

and at $\cos \Theta=0$ :

$$
\tilde{\zeta}_{\mathbf{x}}(z \mid 0)= \pm \frac{\sin (F \pi)}{2 \pi^{\frac{3}{2}}} \frac{\Gamma\left(\frac{3}{2}-z \pm F \mp \frac{1}{2}\right) \Gamma\left(\frac{1}{2}-z \mp F \pm \frac{1}{2}\right)}{\Gamma(z) \Gamma\left(\frac{3}{2}-z\right)} r^{2(z-1)}, \quad \Theta= \pm s \frac{\pi}{2} .
$$


Consequently, we obtain

$$
\tilde{\zeta}_{\mathbf{x}}(0 \mid 0)=0, \quad \mathbf{x} \neq 0
$$

Thus the anomaly is absent everywhere on the plane with the puncture at $\mathbf{x}=0$. This looks rather natural, since twodimensional anomaly density $2 \tilde{\zeta}_{\mathbf{x}}(0 \mid 0)$ is usually identified with quantity $\frac{s}{\pi} \boldsymbol{\partial} \times \mathbf{V}(\mathbf{x})$ [30 32], and the latter quantity in the present case vanishes everywhere on the punctured plane, see Eq.(1.2). We see that the natural anticipations are confirmed provided that the boundary conditions at the puncture are chosen to be physically acceptable, i.e., compatible with the self-adjointness of the Hamiltonianf; we conclude that leakage of the anomaly, in contrast to that of the vacuum condensate or of the vacuum fermion number, does not happen.

We might finish here the discussion of the anomaly problem in the background of a singular magnetic vortex. However, there remains a purely academic question: what is the anomaly density in background (1.1) - (1.2) on the whole plane (without puncturing $\mathbf{x}=0$ )? Just due to a confusion persisting in the literature [33,34, we shall waste now some time to clarify this, otherwise inessential, point.

Background field strength (1.2), when considered on the whole plane, is interpreted in the sense of a distribution (generalized function), i.e., a functional on a set of suitable test functions $f(\mathbf{x})$ :

$$
\int d^{2} x f(\mathbf{x}) \frac{s}{\pi} \boldsymbol{\partial} \times \mathbf{V}(\mathbf{x})=f(0) 2 s \Phi^{(0)}
$$

here $f(\mathbf{x})$ is a continuous function. In particular, choosing $f(\mathbf{x})=1$, one gets

$$
\int d^{2} x \frac{s}{\pi} \boldsymbol{\partial} \times \mathbf{V}(\mathbf{x})=2 s \Phi^{(0)} .
$$

Considering the anomaly density on the whole plane, one is led to study different limiting procedures as $r \rightarrow 0$ and $z \rightarrow 0$ in Eq.(8.23). So, the notorious question is, whether the anomaly density $2 \tilde{\zeta}_{\mathbf{x}}$ can be interpreted in the sense of a distribution which coincides with distribution $\frac{s}{\pi} \boldsymbol{\partial} \times \mathbf{V}(\mathbf{x})$ ? The answer is resolutely negative, and this will be immediately demonstrated below.

First, using explicit form (8.23), we get

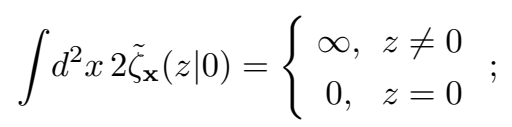

therefore, the anomaly functional cannot be defined on the same set of test functions as that used in Eq.(8.27) (for example, the test functions have to decrease rapidly enough at large (small) distances in the case of $z>0(z<0)$ ). Moreover, if one neglects the requirement of self-consistency, permitting a different (more specified) set of test functions for the anomaly functional, then even this will not save the situation. Let us take $z>0$ for definiteness and use the test functions which are adjusted in such a way that the quantity

$$
\mathcal{A}=\lim _{z \rightarrow 0_{+}} \int d^{2} x f(\mathbf{x}) 2 \tilde{\zeta}_{\mathbf{x}}(z \mid 0)
$$

is finite. Certainly, this quantity can take values in a rather wide range, but it cannot be made equal to the right-hand side of Eq.(8.28). Really, the only source of the dependence on $\Phi^{(0)}$ in the integral in Eq.(8.30) is the factor $\tilde{\zeta}_{\mathbf{x}}(z \mid 0)$, and the latter, as is evident from Eq.(8.23), depends rather on $\left\{\left|\Phi^{(0)}-\Upsilon\right|\right\}$, than on $\Phi^{(0)}$ itself, thus forbidding the linear dependence of $\mathcal{A}$ on $\Phi^{(0)}$. In particular, let us choose test function $f(\mathbf{x})$ in the form

$$
f(\mathbf{x})=\exp \left(-\tilde{\mu}^{2} r^{2}\right)
$$

\footnotetext{
${ }^{4}$ The opposite claim of the authors of Ref. 33] is not justified.
} 
where $\tilde{\mu}$ is the parameter of the dimension of mass. Then, choosing the case of $\cos \Theta=0$ for simplicity and using Eq.(8.25), one gets that Eq.(8.30) takes the form

$$
\mathcal{A}=2\left[s\left(\left\{\left|\Phi^{(0)}-\Upsilon\right|\right\}-\frac{1}{2}\right) \pm \frac{1}{2}\right], \quad \Theta= \pm s \frac{\pi}{2},
$$

which differs clearly from $2 s \Phi^{(0)}$.

Thus, in a singular background the conventional relation between the anomaly density and the background field strength is valid only in the space with punctured singularities. If the singularities are not punctured, then the anomaly density and the background field strength can be interpreted in the sense of distributions, but, in contrast to the assertion of the authors of Refs. [33, 34], the conventional relation is not valid.

\section{ANGULAR MOMENTUM}

Let $\hat{M}$ be an operator in the first-quantized theory, which commutes with the Dirac Hamiltonian

$$
[\hat{M}, H]_{-}=0 .
$$

Then, in the second-quantized theory, the vacuum expectation value of the dynamical variable corresponding to $\hat{M}$ is presented in the form

$$
\mathcal{M}=\int d^{2} x \mathcal{M}_{\mathbf{x}}
$$

where

$$
\mathcal{M}_{\mathbf{x}}=<\operatorname{vac}\left|\frac{1}{2}\left[\Psi^{+}(\mathbf{x}, t), \hat{M} \Psi(\mathbf{x}, t)\right]_{-}\right| \operatorname{vac}>=-\frac{1}{2} \operatorname{tr}<\mathbf{x}|\hat{M} \operatorname{sgn}(H)| \mathbf{x}>.
$$

Commutation relation (9.1) is the evidence of invariance of the theory with $\hat{M}$ being the generator of the symmetry transformations. Since, in the background of a singular magnetic vortex (1.1) - (1.2), there is invariance with respect to rotations around the location of the vortex, one can take $\hat{M}$ as the generator of rotations - the operator of angular momentum in the first-quantized theory (see [17] for more details):

$$
\hat{M}=-i \mathbf{x} \times \boldsymbol{\partial}-\Upsilon+\frac{1}{2} s \beta .
$$

Note that the eigenvalues of operator $\hat{M}$ (9.4) on spinor functions satisfying condition (1.3) are half-integer.

Decomposing Eq.(9.4) into the orbital and spin parts, we get in the second-quantized theory

$$
\mathcal{M}_{\mathbf{x}}=\mathcal{L}_{\mathbf{x}}+\mathcal{S}_{\mathbf{x}}
$$

where

$$
\mathcal{L}_{\mathbf{x}}=\frac{1}{2} \operatorname{tr}<\mathbf{x}|(i \mathbf{x} \times \boldsymbol{\partial}+\Upsilon) \operatorname{sgn}(H)| \mathbf{x}>
$$

and

$$
\mathcal{S}_{\mathbf{x}}=-\frac{1}{4} s \operatorname{tr}<\mathbf{x}|\beta \operatorname{sgn}(H)| \mathbf{x}>.
$$

Since vacuum spin density (9.7) is related to vacuum condensate (7.3),

$$
\mathcal{S}_{\mathbf{x}}=\frac{1}{2} s \mathcal{C}_{\mathbf{x}}
$$


there remains only vacuum orbital angular momentum density (9.6) to be considered.

Let us start, as in Section VII, with the regularized quantity

$$
\mathcal{L}_{\mathbf{x}}(z \mid M)=\frac{1}{2} \operatorname{tr}<\mathbf{x}\left|(i \mathbf{x} \times \boldsymbol{\partial}+\Upsilon) H\left(H^{2}+M^{2}\right)^{-\frac{1}{2}-z}\right| \mathbf{x}>.
$$

The contribution of regular solutions (2.17) and (2.18) to Eq.(9.9) is cancelled upon summation over the sign of energy, whereas the contribution of irregular solution (2.19) to Eq.(9.9) survives:

$$
\begin{gathered}
\mathcal{L}_{\mathbf{x}}(z \mid M)=-\frac{1}{8 \pi} \int_{0}^{\infty} d k k^{2}\left(k^{2}+M^{2}\right)^{-\frac{1}{2}-z}\left\{A ( \frac { k } { \mu } ) ^ { 2 F - 1 } [ L _ { ( + ) } + L _ { ( - ) } ] \left[n_{0} J_{-F}^{2}(k r)+\right.\right. \\
\left.+\left(n_{0}+s\right) J_{1-F}^{2}(k r)\right]+2\left[L_{(+)}-L_{(-)}\right]\left[n_{0} J_{-F}(k r) J_{F}(k r)-\left(n_{0}+s\right) J_{1-F}(k r) J_{-1+F}(k r)\right]+ \\
\left.+A^{-1}\left(\frac{k}{\mu}\right)^{1-2 F}\left[L_{(+)}+L_{(-)}\right]\left[n_{0} J_{F}^{2}(k r)+\left(n_{0}+s\right) J_{-1+F}^{2}(k r)\right]\right\},
\end{gathered}
$$

where $A$ and $L_{( \pm)}$are given by Eqs.(3.5) and (3.6). Transforming the integral in Eq.(9.10) similarly to that as in Appendix D, we get

$$
\mathcal{L}_{\mathbf{x}}(z \mid M)=-\frac{\sin (F \pi)}{2 \pi^{3}} \cos (z \pi) r^{2(z-1)} \int_{|M| r}^{\infty} d w w^{2}\left(w^{2}-M^{2} r^{2}\right)^{-\frac{1}{2}-z} \frac{n_{0} K_{F}^{2}(w)-\left(n_{0}+s\right) K_{1-F}^{2}(w)}{\cosh \left[(2 F-1) \ln \left(\frac{w}{\mu r}\right)+\ln A\right]},
$$

where $\operatorname{Re} z<\frac{1}{2}$. Then we get

$$
\mathcal{L}_{\mathbf{x}} \equiv \mathcal{L}_{\mathbf{x}}(0 \mid 0)=-\frac{\sin (F \pi)}{2 \pi^{3} r^{2}} \int_{0}^{\infty} d w w \frac{n_{0} K_{F}^{2}(w)-\left(n_{0}+s\right) K_{1-F}^{2}(w)}{\cosh \left[(2 F-1) \ln \left(\frac{w}{\mu r}\right)+\ln A\right]} .
$$

Summing Eqs.(9.12) and (9.8), taking into account Eqs.(7.7) and (2.13), we obtain the following expression for the vacuum angular momentum density in the background of a singular magnetic vortex (1.1) - (1.2):

$$
\mathcal{M}_{\mathbf{x}}=\left(\left[\left[\Phi^{(0)}-\Upsilon\right]\right]+\frac{1}{2}\right) \mathcal{N}_{\mathbf{x}},
$$

where vacuum fermion number density $\mathcal{N}_{\mathbf{x}}$ is given by Eq.(3.7). Thus, the total vacuum angular momentum takes the form (see Eq.(3.9))

$$
\mathcal{M}=-\frac{1}{2}\left(\left[\left[\Phi^{(0)}-\Upsilon\right]\right]+\frac{1}{2}\right) \operatorname{sgn}_{0}\left[\left(F-\frac{1}{2}\right) \cos \Theta\right] .
$$

Concluding this section, let us note that relation (9.1) remains to be valid if a constant is added to operator $\hat{M}$. Thus, a definition which is alternative to Eq.(9.4) has been proposed for the angular momentum in the first-quantized theory [35]:

$$
\hat{M}^{\prime}=-i \mathbf{x} \times \boldsymbol{\partial}-\Phi^{(0)}+\frac{1}{2} s \beta .
$$

Then in the second-quantized theory we get

$$
\mathcal{M}_{\mathbf{x}}^{\prime}=-s\left(F-\frac{1}{2}\right) \mathcal{N}_{\mathbf{x}}
$$

and

$$
\mathcal{M}^{\prime}=\frac{1}{2} s\left|F-\frac{1}{2}\right| \operatorname{sgn}_{0}(\cos \Theta) .
$$

Various arguments pro and contra the physical meaningfulness of operator $\hat{M}^{\prime}(9.15)$ are known in the literature (see Refs. [35 37]). However, the crucial point is that operator $\hat{M}$ is the generator of rotations, while operator $\hat{M}^{\prime}$ is not (the eigenvalues of operator $\hat{M}^{\prime}$ on spinor functions are not half-integer). 


\section{SUMMARY AND DISCUSSION}

In the present paper we show that the massless fermionic vacuum under the influence of a singular magnetic vortex (1.1) - (1.2) in 2+1-dimensional space-time attains the following nontrivial characteristics: fermion number (density (3.7) and total (3.9)), energy density (5.2), current (6.14), parity breaking condensate (density (7.7) and total (7.10)) and angular momentum (density (9.13) and total (9.14)). The vacuum spin is related to the vacuum condensate, see Eq.(9.8), the effective potential coincides with the vacuum energy density, see Eq.(5.19), and the parity anomaly is absent, see Eq.(8.8). At large distances from the vortex local vacuum characteristics (densities) are decreasing as inverse powers (with integer exponents in the cases of energy density and current, and with fractional exponents otherwise), see Eqs.(3.8), (5.5), (6.18), and (7.9).

The most general set of boundary conditions at the location of the vortex is used, see Eq.(2.14), providing the self-adjointness of the Dirac Hamiltonian; thus all vacuum polarization effects are depending on self-adjoint extension parameter $\Theta$ or $A$ (3.5). As to the dependence on vortex flux $\Phi^{(0)}$, it has been already anticipated in Introduction that all vacuum polarization effects are gauge invariant and thus depend on reduced vortex flux $\Phi^{(0)}-\Upsilon$ rather than on $\Phi^{(0)}$ or $\Upsilon$ separately. To be more precise, all effects are certainly depending on the fractional part of $\Phi^{(0)}-\Upsilon$ (i.e. on $F(2.15)$ ). It is clear that the dependence on the integer part of $\Phi^{(0)}-\Upsilon$ can be achieved with the help of boundary condition, i.e. choosing the value of $\Theta$ differently for different values of $\left[\left[\Phi^{(0)}-\Upsilon\right]\right.$. However, the vacuum angular momentum, in contrast to all other vacuum characteristics, yields a pattern of other, much more essential, dependence on $\left[\left[\Phi^{(0)}-\Upsilon\right]\right.$. This is owing to the remarkable linear relation between the vacuum angular momentum and fermion number, see Eq.(9.13), which in its turn is due to the choice of the generator of rotations in the capacity of the operator of angular momentum in the first-quantized theory, see Eq.(9.4). Although all vacuum characteristics are vanishing at integer values of $\Phi^{(0)}-\Upsilon$ (i.e. at $F=\frac{1}{2}-\frac{1}{2} s$ ). 5 at noninteger and non-half-integer values (i.e. at $0<F<\frac{1}{2}$ and at $\frac{1}{2}<F<1$ ) the absolute value of the vacuum angular momentum, in contrast to all other vacuum characteristics, is increasing linearly with the increase of the absolute value of $\left[\left[\Phi^{(0)}-\Upsilon\right]\right]$, see Eqs.(9.13) and (9.14). Thus, if the vacuum angular momentum could become somehow detectable, then this would provide us with a unique explicit evidence in favour of physical effects that depend essentially both on integer and fractional parts of the enclosed magnetic flux. Note also that at half-integer values of $\Phi^{(0)}-\Upsilon$ (i.e. at $F=\frac{1}{2}$ ) the vacuum fermion number and angular momentum are vanishing, whereas the vacuum energy density, current and condensate are nonvanishing (the total condensate is even infinite), see Eqs.(5.3), (6.17), (7.8) and (7.10), unless one chooses a certain boundary condition to be discussed immediately below.

Among the whole variety of boundary conditions which are specified by self-adjoint extension parameter $\Theta$, condition $\cos \Theta=0$ ( or $\Theta= \pm \frac{\pi}{2}$ ) is distinguished, since it corresponds to one of the two components of a solution to the Dirac equation being regular for all $n$ : if $\Theta=s \frac{\pi}{2}$, then the lower components are regular, and, if $\Theta=-s \frac{\pi}{2}$, then the upper components are regular, see Eqs. $(2.17)-(2.20)$. This condition is parity invariant, and under it the vacuum fermion number, condensate and angular momentum are vanishing, whereas the vacuum energy density and current are nonvanishing, see Eqs.(5.4) and (6.15). It should be noted that this condition is extensively discussed in the literature, being involved into the two most popular ones: the condition of maximal simplicity [39]

\footnotetext{
${ }^{5}$ This confirms once more the general fact that a singular magnetic vortex is physically unobservable at integer values of $\Phi^{(0)}-\Upsilon[13]$. It was as far back as 1931 that Dirac used actually this fact to obtain his famous condition for the magnetic monopole quantization 38 .
} 


$$
\Theta=\left\{\begin{aligned}
s \frac{\pi}{2}, & s\left(\Phi^{(0)}-\Upsilon\right)>0 \\
-s \frac{\pi}{2}, & s\left(\Phi^{(0)}-\Upsilon\right)<0
\end{aligned}\right\}
$$

and the condition of minimal irregularity 15,18

$$
\Theta=\left\{\begin{array}{cc}
s \frac{\pi}{2}, & -\frac{1}{2}<s\left(\left\{\mid \Phi^{(0)}-\Upsilon\right\}-\frac{1}{2}\right)<0 \\
0, & \left\{\mid \Phi^{(0)}-\Upsilon\right\}=\frac{1}{2} \\
-s \frac{\pi}{2}, & 0<s\left(\left\{\mid \Phi^{(0)}-\Upsilon\right\}-\frac{1}{2}\right)<\frac{1}{2}
\end{array}\right\} ;
$$

here, both in Eqs.(10.1) and(10.2), it is implied that $\left\{\mid \Phi^{(0)}-\Upsilon\right\} \neq 0$.

Under condition (10.1) we get

$$
\mathcal{E}_{\mathbf{x}}^{\text {ren }}=\left\{\begin{array}{cc}
\frac{\tan \left(\left\{\Phi^{(0)}-\Upsilon \mid\right\} \pi\right)}{12 \pi r^{3}}\left\{\left|\Phi^{(0)}-\Upsilon\right|\right\}\left(\frac{1}{4}-\left\{\mid \Phi^{(0)}-\Upsilon\right\}^{2}\right), & \Phi^{(0)}-\Upsilon>0 \\
\frac{\tan \left[\left(1-\left\{\mid \Phi^{(0)}-\Upsilon\right\}\right) \pi\right]}{12 \pi r^{3}}\left(1-\left\{\mid \Phi^{(0)}-\Upsilon\right\}\right)\left[\frac{1}{4}-\left(1-\left\{\left|\Phi^{(0)}-\Upsilon\right|\right\}\right)^{2}\right], & \Phi^{(0)}-\Upsilon<0
\end{array}\right\}
$$

and

$$
j_{\varphi}(\mathbf{x})=\left\{\begin{array}{cc}
-\frac{\tan \left(\left\{\left|\Phi^{(0)}-\Upsilon\right|\right\} \pi\right)}{4 \pi r^{2}}\left(\frac{1}{4}-\left\{\left|\Phi^{(0)}-\Upsilon\right|\right\}^{2}\right), & \Phi^{(0)}-\Upsilon>0 \\
\frac{\tan \left[\left(1-\left\{\left|\Phi^{(0)}-\Upsilon\right|\right\}\right) \pi\right]}{4 \pi r^{2}}\left[\frac{1}{4}-\left(1-\left\{\mid \Phi^{(0)}-\Upsilon\right\}\right)^{2}\right], & \Phi^{(0)}-\Upsilon<0
\end{array}\right\} .
$$

Under condition (10.2) we get

$$
\begin{gathered}
\mathcal{E}_{\mathbf{x}}^{\text {ren }}=\left\{\begin{array}{cc}
\frac{\tan \left(\left\{\Phi^{(0)}-\Upsilon \mid\right\} \pi\right)}{24 \pi r^{3}}\left(\left\{\mid \Phi^{(0)}-\Upsilon\right\}-\frac{1}{2}\right)\left[3\left|\left\{\left|\Phi^{(0)}-\Upsilon\right|\right\}-\frac{1}{2}\right|-\right. & \\
\left.-2\left(\left\{\left|\Phi^{(0)}-\Upsilon\right|\right\}-\frac{1}{2}\right)^{2}-1\right], & \left\{\left|\Phi^{(0)}-\Upsilon\right|\right\} \neq \frac{1}{2} \\
\frac{1}{24 \pi^{2} r^{3}}, & \left\{\Phi^{(0)}-\Upsilon\right\}=\frac{1}{2}
\end{array}\right\}, \\
j_{\varphi}(\mathbf{x})=\left\{\begin{array}{cc}
\frac{\tan \left(\left\{\Phi^{(0)}-\Upsilon \mid\right\} \pi\right)}{4 \pi r^{2}}\left|\left\{\Phi^{(0)}-\Upsilon\right\}-\frac{1}{2}\right|\left(\left|\left\{\left|\Phi^{(0)}-\Upsilon\right|\right\}-\frac{1}{2}\right|-1\right), & \left\{\mid \Phi^{(0)}-\Upsilon\right\} \neq \frac{1}{2} \\
0, & \left\{\mid \Phi^{(0)}-\Upsilon\right\}=\frac{1}{2}
\end{array}\right\}, \\
\mathcal{C}_{\mathbf{x}}=\left\{\begin{array}{cc}
0, & \left\{\mid \Phi^{(0)}-\Upsilon\right\} \neq \frac{1}{2} \\
-\frac{1}{4 \pi^{2} r^{2}}, & \left\{\mid \Phi^{(0)}-\Upsilon\right\}=\frac{1}{2}
\end{array}\right\}
\end{gathered}
$$

and

$$
\mathcal{C}=\left\{\begin{array}{rr}
0, & \left\{\left|\Phi^{(0)}-\Upsilon\right|\right\} \neq \frac{1}{2} \\
-\infty, & \left\{\left|\Phi^{(0)}-\Upsilon\right|\right\}=\frac{1}{2}
\end{array}\right\}
$$

It is clear that Eqs.(10.5) - (10.8), in contrast to Eqs.(10.3) - (10.4), are periodic in the value of the vortex flux, however, they, excepting $\mathcal{E}_{\mathbf{x}}^{\text {ren }}(10.5)$, have discontinuities (even an infinite jump in $\mathcal{C}(10.8)$ ) at both sides of half-integer values of the reduced vortex flux $\left(\left\{\mid \Phi^{(0)}-\Upsilon\right\}=\frac{1}{2}\right)$.

As it should be expected, all vacuum polarization effects are invariant under transitions to equivalent representations of the Clifford algebra (i.e. independent of $\chi_{s}$ ). Under the transition to an inequivalent representation (i.e. under 
$s \rightarrow-s$ ) the vacuum fermion number and angular momentum change their sign, while the vacuum energy density, current and condensate remain unchanged. The latter fact has immediate but far reaching consequences for the more realistic $2+1$-dimensional fermionic model without parity violation.

In this model a quantized spinor field is assigned to a reducible representation composed of two inequivalent irreducible ones. Namely, the Dirac $\gamma$ matrices are chosen in the form

$$
\Gamma^{0}=\left(\begin{array}{cc}
\gamma^{0} & 0 \\
0 & -\gamma^{0}
\end{array}\right), \quad \boldsymbol{\Gamma}=\left(\begin{array}{cc}
\gamma & 0 \\
0 & -\gamma
\end{array}\right),
$$

where $\gamma^{0}$ and $\gamma$ are the $2 \times 2$ matrices of the irreducible representation, see Eqs.(2.7) - (2.9), and a four-component spinor $\Xi$ is a doublet of two-component spinors $\Psi_{+}$and $\Psi_{-}$:

$$
\Xi=\left(\begin{array}{c}
\Psi_{+} \\
\Psi_{-}
\end{array}\right)
$$

Term $\Xi^{+} \Gamma^{0} \Xi$ is invariant under all types of parity transformations (including space and time reflections) but violates chiral symmetry (see, for example, Ref. 40]). Such four-component quantized spinor fields may be relevant for planar condensed matter systems with two sublattices, exhibiting type-II superconductivity [41. Our concern is in the following: how the ground state is affected by an external field configuration in the form of a singular magnetic vortex $(1.1)-(1.2) ?$

Actually, the vacuum polarization effects which are due to $\Psi_{+}$(upper component of doublet (10.10)) have been already determined in the present paper. To determine the vacuum polarization effects which are due to $\Psi_{-}$(lower component of doublet (10.10)), one has to repeat all calculations after changing $\gamma^{0} \rightarrow-\gamma^{0}, \gamma^{1} \rightarrow-\gamma^{1}, \gamma^{2} \rightarrow-\gamma^{2}$, see Eq.(10.9). Doing this, we find that the latter effects are equal to the former ones after changing $s \rightarrow-s$. Thus, polarization of the vacuum for $\Xi$ (10.10) is obtained just by summing polarization of the vacuum for $\Psi$ (2.1) over $s= \pm 1$. In particular, generalized current $\sum_{s= \pm 1} \mathbf{J}^{3}$, in contrast to $\sum_{s= \pm 1} \mathbf{J}$, vanishes identically (see Eq.(8.2)), and the anomaly problem which was worrying us along the whole Section VIII is absent at all. Also in this case the vacuum spin, as well as the vacuum angular momentum and fermion number, vanishes identically (see Eq.(9.8)), whereas the vacuum condensate survives but exhibits chiral symmetry breaking, since it corresponds to the vacuum expectation value of $\Xi^{+} \Gamma^{0} \Xi$. Summarizing, the nontrivial vacuum characteristics are:

energy density

$$
\begin{aligned}
& \sum_{s= \pm 1} \mathcal{E}_{\mathbf{x}}^{\mathrm{ren}}=\frac{\left.\sin \left(\left\{\mid \Phi^{(0)}-\Upsilon\right\}\right\}\right)}{\pi r^{3}}\left\{\frac{\frac{1}{2}-\left\{\left|\Phi^{(0)}-\Upsilon\right|\right\}}{6 \cos \left(\left\{\left|\Phi^{(0)}-\Upsilon\right|\right\} \pi\right)}\left[\frac{3}{4}-\left\{\left|\Phi^{(0)}-\Upsilon\right|\right\}\left(1-\left\{\left|\Phi^{(0)}-\Upsilon\right|\right\}\right)\right]+\right. \\
& \left.+\frac{1}{\pi^{2}} \int_{0}^{\infty} d w w^{2}\left[K_{\left.\left\{\mid \Phi^{(0)}-\Upsilon\right\}\right\}}^{2}(w)-K_{1-\left\{\Phi^{(0)}-\Upsilon \mid\right\}}^{2}(w)\right] \tanh \left[\left(2\left\{\mid \Phi^{(0)}-\Upsilon\right\}-1\right) \ln \left(\frac{w}{\mu r}\right)+\ln \bar{A}\right]\right\},
\end{aligned}
$$

current

$$
\begin{gathered}
\sum_{s= \pm 1} j_{\varphi}(\mathbf{x})=\frac{2 \sin \left(\left\{\left|\Phi^{(0)}-\Upsilon\right|\right\} \pi\right)}{\pi r^{2}}\left\{\frac{\left(\left\{\mid \Phi^{(0)}-\Upsilon\right\}-\frac{1}{2}\right)^{2}}{4 \cos \left(\left\{\mid \Phi^{(0)}-\Upsilon\right\} \pi\right)}-\right. \\
\left.-\frac{1}{\pi^{2}} \int_{0}^{\infty} d w w K_{\left\{\left|\Phi^{(0)}-\Upsilon\right|\right\}}(w) K_{\left.1-\left\{\mid \Phi^{(0)}-\Upsilon\right\}\right\}}(w) \tanh \left[\left(2\left\{\left|\Phi^{(0)}-\Upsilon\right|\right\}-1\right) \ln \left(\frac{w}{\mu r}\right)+\ln \bar{A}\right]\right\}
\end{gathered}
$$


condensate density

$$
\sum_{s= \pm 1} \mathcal{C}_{\mathbf{x}}=-\frac{\sin \left(\left\{\mid \Phi^{(0)}-\Upsilon\{\pi)\right.\right.}{\pi^{3} r^{2}} \int_{0}^{\infty} d w w \frac{K_{\left.\left\{\mid \Phi^{(0)}-\Upsilon\right\}\right\}}^{2}(w)+K_{1-\left\{\Phi^{(0)}-\Upsilon \mid\right\}}^{2}(w)}{\cosh \left[\left(2\left\{\Phi^{(0)}-\Upsilon \mid\right\}-1\right) \ln \left(\frac{w}{\mu r}\right)+\ln \bar{A}\right]}
$$

and condensate total

$$
\sum_{s= \pm 1} \mathcal{C}=-\frac{\operatorname{sgn}_{0}(\cos \Theta)}{2\left|\left\{\left|\Phi^{(0)}-\Upsilon\right|\right\}-\frac{1}{2}\right|}
$$

where

$$
\bar{A}=2^{1-2\left\{\left|\Phi^{(0)}-\Upsilon\right|\right\}} \frac{\Gamma\left(1-\left\{\left|\Phi^{(0)}-\Upsilon\right|\right\}\right)}{\Gamma\left(\left\{\left|\Phi^{(0)}-\Upsilon\right|\right\}\right)} \tan \left(\frac{\Theta}{2}+\frac{\pi}{4}\right) .
$$

It should be noted that chiral symmetry breaking in the background of regular configurations of an external magnetic field has been extensively discussed in the literature [42,43]. We conclude that chiral symmetry breaking occurs also in the background of a singular configuration of an external magnetic field, as a result of leakage of the condensate from the singularity point.

\section{ACKNOWLEDGEMENTS}

I thank H.Leutwyler for interesting discussions. This research was supported by the State Foundation for Fundamental Research of Ukraine (project 2.4/320) and the Swiss National Science Foundation (grant CEEC/NIS/96-98/7 IP 051219).

\section{APPENDIX A}

In view of the discussion in Section IV, we consider here a more general case of massive Hamiltonian $\tilde{H}$ (4.6). The relevant partial Hamiltonian has the form

$$
\tilde{h}_{n_{0}}=\left(\begin{array}{cc}
m & e^{i \chi_{s}}\left[\partial_{r}+(1-F) r^{-1}\right] \\
e^{-i \chi_{s}}\left(-\partial_{r}-F r^{-1}\right) & -m
\end{array}\right) .
$$

Let $\tilde{h}$ be the operator in the form of Eq.(A.1), which acts on the domain of functions $\xi^{0}(r)$ that are regular at $r=0$. Then its adjoint $\tilde{h}^{\dagger}$ which is defined by the relation

$$
\int_{0}^{\infty} d r r\left[\tilde{h}^{\dagger} \xi(r)\right]^{\dagger} \xi^{0}(r)=\int_{0}^{\infty} d r r[\xi(r)]^{\dagger}\left[\tilde{h} \xi^{0}(r)\right]
$$

acts on the domain of functions $\xi(r)$ that are not necessarily regular at $r=0$. So the question is, whether the domain of definition of $\tilde{h}$ can be extended, resulting in both the operator and its adjoint being defined on the same domain of functions? To answer this, one has to construct the eigenspaces of $\tilde{h}^{\dagger}$ with complex eigenvalues. They are spanned by the linearly independent square-integrable solutions correspoding to the pair of purely imaginary eigenvalues,

$$
\tilde{h}^{\dagger} \xi^{ \pm}(r)= \pm i \mu \xi^{ \pm}(r)
$$

where $\mu>0$ is inserted for the dimension reasons. It can be shown that in the case of Eq.(A.1) only one pair of such solutions exists, thus the deficiency index of $\tilde{h}$ is equal to $(1,1)$. This pair is given by the following expression 


$$
\xi^{ \pm}(r)=\frac{1}{N}\left(\begin{array}{l}
e^{i \chi_{s}} \exp \left[ \pm \frac{i}{2} \operatorname{sgn}(m) \eta\right] K_{F}(\tilde{\mu} r) \\
\operatorname{sgn}(m) \exp \left[\mp \frac{i}{2} \operatorname{sgn}(m) \eta\right] K_{1-F}(\tilde{\mu} r)
\end{array}\right)
$$

where $N$ is a certain normalization factor and

$$
\tilde{\mu}=\sqrt{\mu^{2}+m^{2}}, \eta=\arctan \left(\frac{\mu}{|m|}\right) .
$$

Self-adjoint extended operator $\tilde{h}^{\theta_{s}}$ is defined on the domain of functions of the form

$$
\left(\begin{array}{l}
\tilde{f}_{n_{0}} \\
\tilde{g}_{n_{0}}
\end{array}\right)=\xi^{0}+c\left(\xi^{+}+e^{i \theta_{s}} \xi^{-}\right)
$$

where $c$ is a complex parameter and $\theta_{s}$ is a real continuous parameter which depends, in general, on the choice between the two inequivalent representations of the Clifford algebra. Using the asymptotics of the Macdonald function at small values of the variable, we get

$$
\left(\begin{array}{l}
\tilde{f}_{n_{0}} \\
\tilde{g}_{n_{0}}
\end{array}\right) \underset{r \rightarrow 0}{\sim}\left(\begin{array}{l}
e^{i \chi_{s}} \cos \left\{\frac{1}{2}\left[\theta_{s}-\operatorname{sgn}(m) \eta\right]\right\} 2^{F} \Gamma(F)(\tilde{\mu} r)^{-F} \\
\operatorname{sgn}(m) \cos \left\{\frac{1}{2}\left[\theta_{s}+\operatorname{sgn}(m) \eta\right]\right\} 2^{1-F} \Gamma(1-F)(\tilde{\mu} r)^{-1+F}
\end{array}\right),
$$

or

$$
\begin{gathered}
\left\{\tan \left[\frac{1}{2} \theta_{s}-\frac{1}{2} \operatorname{sgn}(m) \eta\right] \sin \eta-\operatorname{sgn}(m) \cos \eta\right\} \lim _{r \rightarrow 0}(\tilde{\mu} r)^{F} \tilde{f}_{n_{0}}= \\
=-e^{i \chi_{s} 2^{2 F-1}} \frac{\Gamma(F)}{\Gamma(1-F)} \lim _{r \rightarrow 0}(\tilde{\mu} r)^{1-F} \tilde{g}_{n_{0}} .
\end{gathered}
$$

Defining new parameter $\Theta$ by means of relation

$$
\tan \left(s \frac{\Theta}{2}+\frac{\pi}{4}\right)=\left\{\tan \left[\frac{1}{2} \theta_{s}-\frac{1}{2} \operatorname{sgn}(m) \eta\right] \sin \eta-\operatorname{sgn}(m) \cos \eta\right\}^{-1}\left(\frac{2 \mu}{\tilde{\mu}}\right)^{2 F-1} \frac{\Gamma(F)}{\Gamma(1-F)}
$$

we get Eq.(2.14).

Certainly, both $\theta_{s}$ and $\Theta$ can be regarded as self-adjoint extension parameters which are to specify the boundary condition at $r=0$. The use of $\Theta$ in this aspect may seem to be more preferable just for the convenience reasons, because Eq.(2.14) looks much simpler than Eq.(A.8). In particular, Eq.(2.14), in contrast to Eq.(A.8), is independent of $m$ and remains explicitly invariant under $s \rightarrow-s(\Theta$ is independent of $s)$.

In the limit of $m \rightarrow \pm 0$ Eq.(A.9) takes the form

$$
\tan \left(s \frac{\Theta}{2}+\frac{\pi}{4}\right)=-\tan \left[\frac{1}{2} \theta_{s}+\frac{1}{4} \operatorname{sgn}(m) \pi\right] 2^{2 F-1} \frac{\Gamma(F)}{\Gamma(1-F)}
$$

then we get

$$
\theta_{s}=s \theta
$$

where $\theta$ is independent of s.

Concluding this appendix, let us note that all characteristics of the massless fermionic vacuum are depending on $A$ (3.5) rather than on $\Theta$ itself, and $A$ is expressed through $\theta$ in the following way

$$
A=-\tan \left[\frac{1}{2} s \theta+\frac{1}{4} \operatorname{sgn}(m) \pi\right]
$$




\section{APPENDIX B}

With the help of relations (see, for example, Ref. [44])

$$
\begin{gathered}
J_{\rho}(i z)=\exp \left(\frac{i}{2} \rho \pi\right) I_{\rho}(z), \quad-\pi<\arg z \leq \frac{\pi}{2}, \\
I_{\rho}(-z)=\exp (i \rho \pi) I_{\rho}(z), \quad K_{\rho}(-z)=\exp (-i \rho \pi) K_{\rho}(z)-i \pi I_{\rho}(z), \quad-\pi<\arg z<0,
\end{gathered}
$$

we get

$$
\begin{aligned}
& J_{\rho}(k r) J_{\tau}(k r)=\frac{1}{2 i \pi}\left\{\exp \left[\frac{i}{2}(\rho-\tau) \pi\right] I_{\rho}(-i k r) K_{\tau}(-i k r)-\right. \\
& -\exp \left[\frac{i}{2}(\tau-\rho) \pi\right] I_{\rho}(i k r) K_{\tau}(i k r)+\exp \left[\frac{i}{2}(\tau-\rho) \pi\right] I_{\tau}(-i k r) K_{\rho}(-i k r)- \\
& \left.-\exp \left[\frac{i}{2}(\rho-\tau) \pi\right] I_{\tau}(i k r) K_{\rho}(i k r)\right\}
\end{aligned}
$$

Then, using Eq.(B.1), we recast Eq.(3.4) into the form

$$
\mathcal{N}_{\mathbf{x}}=\int_{C \subset} d \omega \mathcal{F}_{1}(\omega)
$$

Here $\omega=k^{2}$ is the new variable of integration; contour $C \subset$ circumvents the real positive semiaxis of variable $\omega$, going along it at infinitely small distances from below and above; and the integrand has the form

$$
\begin{aligned}
& \mathcal{F}_{1}(\omega)=\frac{i}{(4 \pi)^{2}}\left\{A ( \frac { \omega } { \mu ^ { 2 } } ) ^ { F - \frac { 1 } { 2 } } [ L _ { ( + ) } + L _ { ( - ) } ] \left[I_{-F}(r \sqrt{-\omega}) K_{F}(r \sqrt{-\omega})+\right.\right. \\
& \left.+I_{1-F}(r \sqrt{-\omega}) K_{1-F}(r \sqrt{-\omega})\right]+\omega^{F}\left[L_{(+)}-L_{(-)}\right](-\omega)^{-F} I_{F}(r \sqrt{-\omega}) K_{F}(r \sqrt{-\omega})+ \\
& +\omega^{-F}\left[L_{(+)}-L_{(-)}\right](-\omega)^{F} I_{-F}(r \sqrt{-\omega}) K_{F}(r \sqrt{-\omega})- \\
& -\omega^{1-F}\left[L_{(+)}-L_{(-)}\right](-\omega)^{-1+F} I_{1-F}(r \sqrt{-\omega}) K_{1-F}(r \sqrt{-\omega})- \\
& -\omega^{-1+F}\left[L_{(+)}-L_{(-)}\right](-\omega)^{1-F} I_{-1+F}(r \sqrt{-\omega}) K_{1-F}(r \sqrt{-\omega})+ \\
& \left.+A^{-1}\left(\frac{\omega}{\mu^{2}}\right)^{\frac{1}{2}-F}\left[L_{(+)}+L_{(-)}\right]\left[I_{F}(r \sqrt{-\omega}) K_{F}(r \sqrt{-\omega})+I_{-1+F}(r \sqrt{-\omega}) K_{1-F}(r \sqrt{-\omega})\right]\right\} .
\end{aligned}
$$

By continuously deforming the contour of integration in the complex $\omega$-plane as is shown in Fig.1, we arrive at the relation

$$
\int_{C \subset} d \omega \mathcal{F}_{1}(\omega)=\int_{C \smile} d \omega \mathcal{F}_{1}(\omega)+\int_{C \unlhd} d \omega \mathcal{F}_{1}(\omega)+\int_{C \curvearrowright} d \omega \mathcal{F}_{1}(\omega) .
$$

The integrals along semicircles $C \smile$ and $C \curvearrowright$ of infinite radii vanish, whereas the integral along the contour circumventing the real negative semiaxis can be represented as

$$
\begin{aligned}
& \int_{C_{\hookrightarrow}} d \omega \mathcal{F}_{1}(\omega)=-\frac{1}{(4 \pi)^{2}} \int_{0}^{\infty} d u \times \\
& \times\left(A\left(\frac{u}{\mu^{2}}\right)^{F-\frac{1}{2}}\left\{e^{i F \pi}\left[R_{(+)}^{(+)}+R_{(-)}^{(+)}\right]+e^{-i F \pi}\left[R_{(+)}^{(-)}+R_{(-)}^{(-)}\right]\right\} \times\right. \\
& \times\left[I_{-F}(r \sqrt{u}) K_{F}(r \sqrt{u})+I_{1-F}(r \sqrt{u}) K_{1-F}(r \sqrt{u})\right]- \\
& -\left\{e^{i\left(F-\frac{1}{2}\right) \pi}\left[R_{(+)}^{(+)}-R_{(-)}^{(+)}\right]-e^{-i\left(F-\frac{1}{2}\right) \pi}\left[R_{(+)}^{(-)}-R_{(-)}^{(-)}\right]\right\} \times
\end{aligned}
$$




$$
\begin{aligned}
& \times\left[I_{F}(r \sqrt{u}) K_{F}(r \sqrt{u})+I_{-1+F}(r \sqrt{u}) K_{1-F}(r \sqrt{u})\right]+ \\
& +\left\{e^{-i\left(F-\frac{1}{2}\right) \pi}\left[R_{(+)}^{(+)}-R_{(-)}^{(+)}\right]-e^{i\left(F-\frac{1}{2}\right) \pi}\left[R_{(+)}^{(-)}-R_{(-)}^{(-)}\right]\right\} \times \\
& \times\left[I_{-F}(r \sqrt{u}) K_{F}(r \sqrt{u})+I_{1-F}(r \sqrt{u}) K_{1-F}(r \sqrt{u})\right]- \\
& -A^{-1}\left(\frac{u}{\mu^{2}}\right)^{\frac{1}{2}-F}\left\{e^{-i F \pi}\left[R_{(+)}^{(+)}+R_{(-)}^{(+)}\right]+e^{i F \pi}\left[R_{(+)}^{(-)}+R_{(-)}^{(-)}\right]\right\} \times \\
& \left.\times\left[I_{F}(r \sqrt{u}) K_{F}(r \sqrt{u})+I_{-1+F}(r \sqrt{u}) K_{1-F}(r \sqrt{u})\right]\right)
\end{aligned}
$$

where

$$
R_{( \pm)}^{(+)}=2^{-1}\left(\cos (F \pi) \pm \cosh \left\{\left(F-\frac{1}{2}\right)\left[\ln \left(\frac{u}{\mu^{2}}\right)+i \pi\right]+\ln A\right\}\right)^{-1}
$$

and

$$
R_{( \pm)}^{(-)}=2^{-1}\left(\cos (F \pi) \mp \cosh \left\{\left(F-\frac{1}{2}\right)\left[\ln \left(\frac{u}{\mu^{2}}\right)-i \pi\right]+\ln A\right\}\right)^{-1}
$$

Expression (B.5) can be reduced to the form

$$
\begin{aligned}
& \int_{C \leftrightarrows} d \omega \mathcal{F}_{1}(\omega)=-\frac{\sin (F \pi)}{8 \pi^{3}} \int_{0}^{\infty} d u\left\{A\left(\frac{u}{\mu^{2}}\right)^{F-\frac{1}{2}}\left[e^{i F \pi}\left(R_{(+)}^{(+)}+R_{(-)}^{(+)}\right)+e^{-i F \pi}\left(R_{(+)}^{(-)}+R_{(-)}^{(-)}\right)\right]+\right. \\
& \left.+e^{-i\left(F-\frac{1}{2}\right) \pi}\left(R_{(+)}^{(+)}-R_{(-)}^{(+)}\right)-e^{i\left(F-\frac{1}{2}\right) \pi}\left(R_{(+)}^{(-)}-R_{(-)}^{(-)}\right)\right\} K_{F}^{2}(r \sqrt{u})+ \\
& +\frac{\sin (F \pi)}{8 \pi^{3}} \int_{0}^{\infty} d u\left\{e^{i\left(F-\frac{1}{2}\right) \pi}\left(R_{(+)}^{(+)}-R_{(-)}^{(+)}\right)-e^{-i\left(F-\frac{1}{2}\right) \pi}\left(R_{(+)}^{(-)}-R_{(-)}^{(-)}\right)+\right. \\
& \left.+A^{-1}\left(\frac{u}{\mu^{2}}\right)^{\frac{1}{2}-F}\left[e^{-i F \pi}\left(R_{(+)}^{(+)}+R_{(-)}^{(+)}\right)+e^{i F \pi}\left(R_{(+)}^{(-)}+R_{(-)}^{(-)}\right)\right]\right\} K_{1-F}^{2}(r \sqrt{u}) .
\end{aligned}
$$

After further simplifications we arrive at Eq.(3.7) where variable $w=r \sqrt{u}$ is introduced.

\section{APPENDIX C}

As in the previous appendix, with the use of Eq.(B.1), expressions (4.17) and (4.18) are recast into the form

$$
\left[\zeta_{\mathbf{x}}(z \mid m)\right]_{\mathrm{REG}}=\int_{C_{\complement}} d \omega \mathcal{F}_{2}(\omega)
$$

and

$$
\left[\zeta_{\mathbf{x}}(z \mid m)\right]_{\mathrm{IRREG}}=\int_{C \subset} d \omega \mathcal{F}_{3}(\omega),
$$

where

$$
\mathcal{F}_{2}(\omega)=\frac{1}{i(2 \pi)^{2}} \frac{\left(\omega+m^{2}\right)^{1-z}}{(z-1) \omega}\left[F I_{F}(r \sqrt{-\omega}) K_{F}(r \sqrt{-\omega})+(1-F) I_{1-F}(r \sqrt{-\omega}) K_{1-F}(r \sqrt{-\omega})\right]
$$

and 


$$
\begin{aligned}
& \mathcal{F}_{3}(\omega)=\frac{1}{i 2(2 \pi)^{2}}\left(\omega+m^{2}\right)^{-\frac{1}{2}-z}\left\{A \mu^{1-2 F} \omega^{F}\left[\tilde{L}_{(+)}-\tilde{L}_{(-)}\right] I_{-F}(r \sqrt{-\omega}) K_{F}(r \sqrt{-\omega})+\right. \\
& +A \mu^{1-2 F} \omega^{-1+F}\left[\left(m-\sqrt{\omega+m^{2}}\right)^{2} \tilde{L}_{(+)}-\left(m+\sqrt{\omega+m^{2}}\right)^{2} \tilde{L}_{(-)}\right] I_{1-F}(r \sqrt{-\omega}) K_{1-F}(r \sqrt{-\omega})+ \\
& +\omega^{F}\left[\left(m+\sqrt{\omega+m^{2}}\right) \tilde{L}_{(+)}-\left(m-\sqrt{\omega+m^{2}}\right) \tilde{L}_{(-)}\right](-\omega)^{-F} I_{F}(r \sqrt{-\omega}) K_{F}(r \sqrt{-\omega})+ \\
& +\omega^{-F}\left[\left(m+\sqrt{\omega+m^{2}}\right) \tilde{L}_{(+)}-\left(m-\sqrt{\omega+m^{2}}\right) \tilde{L}_{(-)}\right](-\omega)^{F} I_{-F}(r \sqrt{-\omega}) K_{F}(r \sqrt{-\omega})+ \\
& +\omega^{1-F}\left[\left(m-\sqrt{\omega+m^{2}}\right) \tilde{L}_{(+)}-\left(m+\sqrt{\omega+m^{2}}\right) \tilde{L}_{(-)}\right](-\omega)^{-1+F} I_{1-F}(r \sqrt{-\omega}) K_{1-F}(r \sqrt{-\omega})+ \\
& +\omega^{-1+F}\left[\left(m-\sqrt{\omega+m^{2}}\right) \tilde{L}_{(+)}-\left(m+\sqrt{\omega+m^{2}}\right) \tilde{L}_{(-)}\right](-\omega)^{1-F} I_{-1+F}(r \sqrt{-\omega}) K_{1-F}(r \sqrt{-\omega})+ \\
& +A^{-1} \mu^{2 F-1} \omega^{-F}\left[\left(m+\sqrt{\omega+m^{2}}\right)^{2} \tilde{L}_{(+)}-\left(m-\sqrt{\omega+m^{2}}\right)^{2} \tilde{L}_{(-)}\right] I_{F}(r \sqrt{-\omega}) K_{F}(r \sqrt{-\omega})+ \\
& \left.+A^{-1} \mu^{2 F-1} \omega^{1-F}\left[\tilde{L}_{(+)}-\tilde{L}_{(-)}\right] I_{-1+F}(r \sqrt{-\omega}) K_{1-F}(r \sqrt{-\omega})\right\} .
\end{aligned}
$$

Deforming the contour of integration in Eqs.(C.1) and (C.2) as is shown in Figs. 2 and 3 correspondingly, we arrive at the relation

$$
\int_{C \complement} d \omega \mathcal{F}_{j}(\omega)=\int_{C \smile} d \omega \mathcal{F}_{j}(\omega)+\int_{C \unlhd} d \omega \mathcal{F}_{j}(\omega)+\int_{C \curvearrowright} d \omega \mathcal{F}_{j}(\omega)+\int_{C_{\bigcirc}} d \omega \mathcal{F}_{j}(\omega), \quad j=2,3 .
$$

Here both integrands (C.3) and (C.4) have a branching point at $\omega=-m^{2}$ (therefore a cut is drawn from $-m^{2}$ to $-\infty)$ and a pole on the real axis: at $\omega=0$ in the case of Eq.(C.3), and at $\omega=-\kappa^{2}$ in the case of Eq.(C.4), where $\kappa^{2}=m^{2}-E_{\mathrm{BS}}^{2}$ and $E_{\mathrm{BS}}$ is determined by Eq.(4.14). At $1<\operatorname{Re} z<2$ in the case of $\mathcal{F}_{2}(\omega)$ and at $\frac{1}{2}<\operatorname{Re} z<1$ in the case of $\mathcal{F}_{3}(\omega)$, the integrals along semicircles $C \smile$ and $C_{\curvearrowright}$ of infinite radii vanish, whereas the integrals along the contour circumventing the cut on the real negative axis can be represented as

$$
\begin{aligned}
& \int_{C \leftrightarrows} d \omega \mathcal{F}_{2}(\omega)=\frac{\sin (z \pi)}{2 \pi^{2}(z-1)} \times \\
& \times \int_{m^{2}}^{\infty} \frac{d u}{u}\left(u-m^{2}\right)^{1-z}\left[F I_{F}(r \sqrt{u}) K_{F}(r \sqrt{u})+(1-F) I_{1-F}(r \sqrt{u}) K_{1-F}(r \sqrt{u})\right]
\end{aligned}
$$

and

$$
\begin{aligned}
& \int_{C \hookrightarrow} d \omega \mathcal{F}_{3}(\omega)=\frac{1}{2(2 \pi)^{2}} \int_{m^{2}}^{\infty} d u\left(u-m^{2}\right)^{-\frac{1}{2}-z} \times \\
& \times\left(A \mu^{1-2 F} u^{F}\left\{e^{i(F-z) \pi}\left[\tilde{R}_{(+)}^{(+)}-\tilde{R}_{(-)}^{(+)}\right]-e^{-i(F-z) \pi}\left[\tilde{R}_{(+)}^{(-)}-\tilde{R}_{(-)}^{(-)}\right]\right\} I_{-F}(r \sqrt{u}) K_{F}(r \sqrt{u})-\right. \\
& -A \mu^{1-2 F} u^{-1+F}\left\{e^{i(F-z) \pi}\left[\left(m-i \sqrt{u-m^{2}}\right)^{2} \tilde{R}_{(+)}^{(+)}-\left(m+i \sqrt{u-m^{2}}\right)^{2} \tilde{R}_{(-)}^{(+)}\right]-\right. \\
& \left.-e^{-i(F-z) \pi}\left[\left(m-i \sqrt{u-m^{2}}\right)^{2} \tilde{R}_{(+)}^{(-)}-\left(m+i \sqrt{u-m^{2}}\right)^{2} \tilde{R}_{(-)}^{(-)}\right]\right\} I_{1-F}(r \sqrt{u}) K_{1-F}(r \sqrt{u})+ \\
& +\left\{e^{i(F-z)}\left[\left(m+i \sqrt{u-m^{2}}\right) \tilde{R}_{(+)}^{(+)}-\left(m-i \sqrt{u-m^{2}}\right) \tilde{R}_{(-)}^{(+)}\right]-\right. \\
& \left.-e^{-i(F-z) \pi}\left[\left(m+i \sqrt{u-m^{2}}\right) \tilde{R}_{(+)}^{(-)}-\left(m-i \sqrt{u-m^{2}}\right) \tilde{R}_{(-)}^{(-)}\right]\right\} I_{F}(r \sqrt{u}) K_{F}(r \sqrt{u})+ \\
& +\left\{e^{-i(F+z) \pi}\left[\left(m+i \sqrt{u-m^{2}}\right) \tilde{R}_{(+)}^{(+)}-\left(m-i \sqrt{u-m^{2}}\right) \tilde{R}_{(-)}^{(+)}\right\}-\right. \\
& \left.-e^{i(F+z) \pi}\left[\left(m+i \sqrt{u-m^{2}}\right) \tilde{R}_{(+)}^{(-)}-\left(m-i \sqrt{u-m^{2}}\right) \tilde{R}_{(-)}^{(-)}\right]\right\} I_{-F}(r \sqrt{u}) K_{F}(r \sqrt{u})- \\
& -\left\{e^{-i(F+z) \pi}\left[\left(m-i \sqrt{u-m^{2}}\right) \tilde{R}_{(+)}^{(+)}-\left(m+i \sqrt{u-m^{2}}\right) \tilde{R}_{(-)}^{(+)}\right]-\right. \\
& \left.-e^{i(F+z) \pi}\left[\left(m-i \sqrt{u-m^{2}}\right) \tilde{R}_{(+)}^{(-)}-\left(m+i \sqrt{u-m^{2}}\right) \tilde{R}_{(-)}^{(-)}\right]\right\} I_{1-F}(r \sqrt{u}) K_{1-F}(r \sqrt{u})- \\
& -\left\{e^{i(F-z) \pi}\left[\left(m-i \sqrt{u-m^{2}}\right) \tilde{R}_{(+)}^{(+)}-\left(m+i \sqrt{u-m^{2}}\right) \tilde{R}_{(-)}^{(+)}\right]-\right.
\end{aligned}
$$




$$
\begin{aligned}
& \left.-e^{-i(F-z) \pi}\left[\left(m-i \sqrt{u-m^{2}}\right) \tilde{R}_{(+)}^{(-)}-\left(m+i \sqrt{u-m^{2}}\right) \tilde{R}_{(-)}^{(-)}\right]\right\} I_{-1+F}(r \sqrt{u}) K_{1-F}(r \sqrt{u})+ \\
& +A^{-1} \mu^{2 F-1} u^{-F}\left\{e^{-i(F+z) \pi}\left[\left(m+i \sqrt{u-m^{2}}\right)^{2} \tilde{R}_{(+)}^{(+)}-\left(m-i \sqrt{u-m^{2}}\right)^{2} \tilde{R}_{(-)}^{(+)}\right]-\right. \\
& \left.-e^{i(F+z) \pi}\left[\left(m+i \sqrt{u-m^{2}}\right)^{2} \tilde{R}_{(+)}^{(-)}-\left(m-i \sqrt{u-m^{2}}\right)^{2} \tilde{R}_{(-)}^{(-)}\right]\right\} I_{F}(r \sqrt{u}) K_{F}(r \sqrt{u})- \\
& \left.-A^{-1} \mu^{2 F-1} u^{1-F}\left\{e^{-i(F+z) \pi}\left[\tilde{R}_{(+)}^{(+)}-\tilde{R}_{(-)}^{(+)}\right]-e^{i(F+z) \pi}\left[\tilde{R}_{(+)}^{(-)}-\tilde{R}_{(-)}^{(-)}\right]\right\} I_{-1+F}(r \sqrt{u}) K_{1-F}(r \sqrt{u})\right),
\end{aligned}
$$

where

$$
\begin{aligned}
& \tilde{R}_{( \pm)}^{(+)}=\left[A \mu^{1-2 F} u^{-1+F} e^{i F \pi}\left(m \mp i \sqrt{u-m^{2}}\right)+\right. \\
& \left.+2 \cos (F \pi)+A^{-1} \mu^{2 F-1} u^{-F} e^{-i F \pi}\left(m \pm i \sqrt{u-m^{2}}\right)\right]^{-1}
\end{aligned}
$$

and

$$
\begin{aligned}
& \tilde{R}_{( \pm)}^{(-)}=\left[A \mu^{1-2 F} u^{-1+F} e^{-i F \pi}\left(m \mp i \sqrt{u-m^{2}}\right)+\right. \\
& \left.+2 \cos (F \pi)+A^{-1} \mu^{2 F-1} u^{-F} e^{i F \pi}\left(m \pm i \sqrt{u-m^{2}}\right)\right]^{-1} .
\end{aligned}
$$

Adding the integral along the contour enclosing the pole of $\mathcal{F}_{2}(\omega)$

$$
\int_{C_{\bigcirc}} d \omega \mathcal{F}_{2}(\omega)=2 \pi i \operatorname{Res}_{\omega=0} \mathcal{F}_{2}(\omega)
$$

to Eq.(C.6), we arrive at Eq.(4.21) where variable $w=r \sqrt{u}$ is introduced.

As to expression (C.7), it can be simplified

$$
\begin{aligned}
& \int_{C_{\hookrightarrow}} d \omega \mathcal{F}_{3}(\omega)=\frac{\sin (z \pi)}{2 \pi^{2}} \int_{m^{2}}^{\infty} d u\left(u-m^{2}\right)^{-z}\left[I_{F}(r \sqrt{u}) K_{F}(r \sqrt{u})+I_{1-F}(r \sqrt{u}) K_{1-F}(r \sqrt{u})\right]+ \\
& +\frac{\sin (F \pi)}{4 \pi^{3}} \int_{m^{2}}^{\infty} d u\left(u-m^{2}\right)^{-\frac{1}{2}-z}\left(e ^ { - i z \pi } \left\{\left[A \mu^{1-2 F} u^{F} e^{i F \pi}+\left(m+i \sqrt{u-m^{2}}\right) e^{-i F \pi}\right] \tilde{R}_{(+)}^{(+)}-\right.\right. \\
& \left.-\left[A \mu^{1-2 F} u^{F} e^{i F \pi}+\left(m-i \sqrt{u-m^{2}}\right) e^{-i F \pi}\right] \tilde{R}_{(-)}^{(+)}\right\}-e^{i z \pi}\left\{\left[A \mu^{1-2 F} u^{F} e^{-i F \pi}+\right.\right. \\
& \left.\left.\left.+\left(m+i \sqrt{u-m^{2}}\right) e^{i F \pi}\right] \tilde{R}_{(+)}^{(-)}-\left[A \mu^{1-2 F} u^{F} e^{-i F \pi}+\left(m-i \sqrt{u-m^{2}}\right) e^{i F \pi}\right] \tilde{R}_{(-)}^{(-)}\right\}\right) K_{F}^{2}(r \sqrt{u})- \\
& -\frac{\sin (F \pi)}{4 \pi^{3}} \int_{m^{2}}^{\infty} d u\left(u-m^{2}\right)^{-\frac{1}{2}-z}\left(e ^ { - i z \pi } \left\{\left[A^{-1} \mu^{2 F-1} u^{1-F} e^{-i F \pi}+\left(m-i \sqrt{u-m^{2}}\right) e^{i F \pi}\right] \tilde{R}_{(+)}^{(+)}-\right.\right. \\
& \left.-\left[A^{-1} \mu^{2 F-1} u^{1-F} e^{-i F \pi}+\left(m+i \sqrt{u-m^{2}}\right) e^{i F \pi}\right] \tilde{R}_{(-)}^{(+)}\right\}- \\
& -e^{i z \pi}\left\{\left[A^{-1} \mu^{2 F-1} u^{1-F} e^{i F \pi}+\left(m-i \sqrt{u-m^{2}}\right) e^{-i F \pi}\right] \tilde{R}_{(+)}^{(-)}-\right. \\
& \left.\left.-\left[A^{-1} \mu^{2 F-1} u^{1-F} e^{i F \pi}+\left(m+i \sqrt{u-m^{2}}\right) e^{-i F \pi}\right] \tilde{R}_{(-)}^{(-)}\right\}\right) K_{1-F}^{2}(r(\sqrt{u}) .
\end{aligned}
$$

Simplifying further the last expression, we arrive at the terms in relation (4.22) that are represented as integrals over variable $w=r \sqrt{u}$.

It remains to consider the integral along the contour enclosing the pole of $\mathcal{F}_{3}(\omega)$

$$
\int_{C_{\bigcirc}} d \omega \mathcal{F}_{3}(\omega)=2 \pi i \operatorname{Res}_{\omega=-\kappa^{2}} \mathcal{F}_{3}(\omega) .
$$


Choosing the branch for fractional exponents according to the prescription

$$
\left(-\kappa^{2}\right)^{\rho}=\kappa^{2 \rho} e^{i \rho \pi}, \quad 0<\rho<1
$$

we get

$$
\begin{aligned}
& \int_{C_{\mathrm{O}}} d \omega \mathcal{F}_{3}(\omega)= \pm \frac{1}{4 \pi}\left|E_{\mathrm{BS}}\right|^{-1-2 z}\left[A \mu^{1-2 F} \kappa^{2 F} e^{i F \pi} I_{-F}(\kappa r) K_{F}(\kappa r)-\right. \\
& -A \mu^{1-2 F} \kappa^{-2(1-F)} e^{i F \pi}\left(m \mp\left|E_{\mathrm{BS}}\right|\right)^{2} I_{1-F}(\kappa r) K_{1-F}(\kappa r)+e^{i F \pi}\left(m \pm\left|E_{\mathrm{BS}}\right|\right) I_{F}(\kappa r) K_{F}(\kappa r)+ \\
& +e^{-i F \pi}\left(m \pm\left|E_{\mathrm{BS}}\right|\right) I_{-F}(\kappa r) K_{F}(\kappa r)-e^{-i F \pi}\left(m \mp\left|E_{\mathrm{BS}}\right|\right) I_{1-F}(\kappa r) K_{1-F}(\kappa r)- \\
& -e^{i F \pi}\left(m \mp\left|E_{\mathrm{BS}}\right|\right) I_{-1+F}(\kappa r) K_{1-F}(\kappa r)+A^{-1} \mu^{2 F-1} \kappa^{-2 F} e^{-i F \pi}\left(m \pm\left|E_{\mathrm{BS}}\right|\right)^{2} I_{F}(\kappa r) K_{F}(\kappa r)- \\
& \left.-A^{-1} \mu^{2 F-1} \kappa^{2(1-F)} e^{-i F \pi} I_{-1+F}(\kappa r) K_{1-F}(\kappa r)\right] \underset{\omega=-\kappa^{2}}{\operatorname{Res}_{( \pm)} \tilde{L}_{( \pm)}=} \\
& =\mp \frac{i \sin ^{2}(F \pi)}{\pi^{2}}\left|E_{\mathrm{BS}}\right|^{-1-2 z}\left[\left(m \pm\left|E_{\mathrm{BS}}\right|\right) K_{F}^{2}(\kappa r)+\left(m \mp\left|E_{\mathrm{BS}}\right|\right) K_{1-F}^{2}(\kappa r)\right] \operatorname{Res}_{\omega=-\kappa^{2}} \tilde{L}_{( \pm)},
\end{aligned}
$$

$E_{\mathrm{BS}} \gtrless 0$.

Taking into account relation

$$
\operatorname{Res}_{\omega=-\kappa^{2}} \tilde{L}_{( \pm)}=\frac{1}{i \sin (F \pi)} \frac{\left|E_{\mathrm{BS}}\right| \kappa^{2}}{\left|E_{\mathrm{BS}}\right|(2 F-1) \pm m}, \quad E_{\mathrm{BS}} \gtrless 0,
$$

we arrive at the last term in relation (4.22). Naturally, the result will be the same, if the branch for fractional exponents is chosen alternatively as

$$
\left(-\kappa^{2}\right)^{\rho}=\kappa^{2 \rho} e^{-i \rho \pi}, \quad 0<\rho<1 .
$$

\section{APPENDIX D}

Expression (4.30) is recast into the form

$$
\left[\zeta_{\mathbf{x}}(z \mid M)\right]_{\mathrm{IRREG}}=\int_{C_{\complement}} d \omega \mathcal{F}_{4}(\omega),
$$

where

$$
\begin{aligned}
& \mathcal{F}_{4}(\omega)=\frac{1}{i 2(2 \pi)^{2}}\left(\omega+M^{2}\right)^{-z}\left\{A ( \frac { \omega } { \mu ^ { 2 } } ) ^ { F - \frac { 1 } { 2 } } [ L _ { ( + ) } - L _ { ( - ) } ] \left[I_{-F}(r \sqrt{-\omega}) K_{F}(r \sqrt{-\omega})+\right.\right. \\
& \left.+I_{1-F}(r \sqrt{-\omega}) K_{1-F}(r \sqrt{-\omega})\right]+\omega^{F}\left[L_{(+)}+L_{(-)}\right](-\omega)^{-F} I_{F}(r \sqrt{-\omega}) K_{F}(r \sqrt{-\omega})+\omega^{-F}\left[L_{(+)}+L_{(-)}\right] \times \\
& \times(-\omega)^{F} I_{-F}(r \sqrt{-\omega}) K_{F}(r \sqrt{-\omega})-\omega^{1-F}\left[L_{(+)}+L_{(-)}\right](-\omega)^{-1+F} I_{1-F}(r \sqrt{-\omega}) K_{1-F}(r \sqrt{-\omega})- \\
& -\omega^{-1+F}\left[L_{(+)}+L_{(-)}\right](-\omega)^{1-F} I_{-1+F}(r \sqrt{-\omega}) K_{1-F}(r \sqrt{-\omega})+ \\
& \left.+A^{-1}\left(\frac{\omega}{\mu^{2}}\right)^{\frac{1}{2}-F}\left[L_{(+)}-L_{(-)}\right]\left[I_{F}(r \sqrt{-\omega}) K_{F}(r \sqrt{-\omega})+I_{-1+F}(r \sqrt{-\omega}) K_{1-F}(r \sqrt{-\omega})\right]\right\} .
\end{aligned}
$$

Deforming the contour of integration in Eq.(D.1), we arrive at the same relation as Eq.(B.4), but with contour $C \hookrightarrow$ circumventing the cut from $-M^{2}$ to $-\infty$ (see Fig.4). At $\frac{1}{2}<\operatorname{Re} z<1$ the integrals along semicircles $C \smile$ and $C \curvearrowright$ of infinite radii vanish, so there remains the integral: 


$$
\begin{aligned}
& \int_{{ }_{\hookrightarrow}} d \omega \mathcal{F}_{4}(\omega)=\frac{1}{2(2 \pi)^{2}} \int_{M^{2}}^{\infty} d u\left(u-M^{2}\right)^{-z}\left(A ( \frac { u } { \mu ^ { 2 } } ) ^ { F - \frac { 1 } { 2 } } \left\{e^{i(F-z) \pi}\left[R_{(+)}^{(+)}-R_{(-)}^{(+)}\right]-\right.\right. \\
& \left.-e^{-i(F-z) \pi}\left[R_{(+)}^{(-)}-R_{(-)}^{(-)}\right]\right\}\left[I_{-F}(r \sqrt{u}) K_{F}(r \sqrt{u})+I_{1-F}(r \sqrt{u}) K_{1-F}(r \sqrt{u})\right]- \\
& -\left\{e^{i\left(F-\frac{1}{2}-z\right) \pi}\left[R_{(+)}^{(+)}+R_{(-)}^{(+)}\right]+e^{-i\left(F-\frac{1}{2}-z\right) \pi}\left[R_{(+)}^{(-)}+R_{(-)}^{(-)}\right]\right\}\left[I_{F}(r \sqrt{u}) K_{F}(r \sqrt{u})+\right. \\
& \left.+I_{-1+F}(r \sqrt{u}) K_{1-F}(r \sqrt{u})\right]+\left\{e^{-i\left(F-\frac{1}{2}+z\right) \pi}\left[R_{(+)}^{(+)}+R_{(-)}^{(+)}\right]+e^{i\left(F-\frac{1}{2}+z\right) \pi}\left[R_{(+)}^{(-)}+R_{(-)}^{(-)}\right]\right\} \times \\
& \times\left[I_{-F}(r \sqrt{u}) K_{F}(r \sqrt{u})+I_{1-F}(r \sqrt{u}) K_{1-F}(r \sqrt{u})\right]-A^{-1}\left(\frac{u}{\mu^{2}}\right)^{\frac{1}{2}-F}\left\{e^{-i(F+z) \pi}\left[R_{(+)}^{(+)}-R_{(-)}^{(+)}\right]-\right. \\
& \left.\left.-e^{i(F+z) \pi}\left[R_{(+)}^{(-)}-R_{(-)}^{(-)}\right]\right\}\left[I_{F}(r \sqrt{u}) K_{F}(r \sqrt{u})+I_{-1+F}(r \sqrt{u}) K_{1-F}(r \sqrt{u})\right]\right),
\end{aligned}
$$

where $R_{( \pm)}^{(+)}$and $R_{( \pm)}^{(-)}$are given by Eqs.(B.6) and (B.7). Expression (D.3) can be reduced to the form

$$
\begin{aligned}
& \int_{C} d \omega \mathcal{F}_{4}(\omega)=\frac{\sin (z \pi)}{2 \pi^{2}} \int_{M^{2}}^{\infty} d u\left(u-M^{2}\right)^{-z}\left[I_{F}(r \sqrt{u}) K_{F}(r \sqrt{u})+I_{1-F}(r \sqrt{u}) K_{1-F}(r \sqrt{u})\right]+ \\
& +\frac{\sin (F \pi)}{4 \pi^{3}} \int_{M^{2}}^{\infty} d u\left(u-M^{2}\right)^{-z}\left\{A\left(\frac{u}{\mu^{2}}\right)^{F-\frac{1}{2}}\left[e^{i(F-z) \pi}\left(R_{(+)}^{(+)}-R_{(-)}^{(+)}\right)-e^{-i(F-z) \pi}\left(R_{(+)}^{(-)}-R_{(-)}^{(-)}\right)\right]+\right. \\
& \left.+e^{-i\left(F-\frac{1}{2}+z\right) \pi}\left(R_{(+)}^{(+)}+R_{(-)}^{(+)}\right)+e^{i\left(F-\frac{1}{2}+z\right) \pi}\left(R_{(+)}^{(-)}+R_{(-)}^{(-)}\right)\right\} K_{F}^{2}(r \sqrt{u})- \\
& -\frac{\sin (F \pi)}{4 \pi^{3}} \int_{M^{2}}^{\infty} d u\left(u-M^{2}\right)^{-z}\left\{e^{i\left(F-\frac{1}{2}-z\right) \pi}\left(R_{(+)}^{(+)}+R_{(-)}^{(+)}\right)+e^{-i\left(F-\frac{1}{2}-z\right) \pi}\left(R_{(+)}^{(-)}+R_{(-)}^{(-)}\right)+\right. \\
& \left.+A^{-1}\left(\frac{u}{\mu^{2}}\right)^{\frac{1}{2}-F}\left[e^{-i(F+z) \pi}\left(R_{(+)}^{(+)}-R_{(-)}^{(+)}\right)-e^{i(F+z) \pi}\left(R_{(+)}^{(-)}-R_{(-)}^{(-)}\right)\right]\right\} K_{1-F}^{2}(r \sqrt{u}) .
\end{aligned}
$$

After further simplifications we arrive at Eq.(4.31) where variable $w=r \sqrt{u}$ is introduced.

[1] H.Bethe and R.Peierls, Proc.Roy.Soc.(London) A148, 146 (1935).

[2] L.H.Thomas, Phys.Rev. 47, 903 (1935).

[3] E.Fermi, Ricerca Scientifica 7, 13 (1936).

[4] S.Albeverio, F.Gesztesy, R.Hoegh-Krohn and H. Holden, Solvable Models in Quantum Mechanics (Springer-Verlag, Berlin, 1988).

[5] F.A.Berezin and L.D.Faddeev, Sov.Math.Dokl. 2, 372 (1961).

[6] R.Jackiw, in M.A.B. Beg Memorial Volume, edited by A.Ali and P.Hoodbhoy (World Scientific, Singapore, 1991).

[7] A.S.Goldhaber, Phys.Rev. D16, 1815 (1977).

[8] C.J.Callias, Phys.Rev. D16, 3068 (1977).

[9] E.Witten, Phys.Let. 86B, 283 (1979).

[10] B.Grossman, Phys.Rev.Lett. 50, 464 (1983). 
[11] H.Yamagishi, Phys.Rev. D27, 2383; D28, 977 (1983).

[12] W.Ehrenberg and R.Siday, Proc.Phys.Soc.(London) B62, 8 (1949).

[13] Y.Aharonov and D.Bohm, Phys.Rev. 115, 485 (1959).

[14] Yu.A.Sitenko, Sov.J.Nucl.Phys. 47, 184 (1988).

[15] Yu.A.Sitenko, Nucl.Phys. B342, 655 (1990); Phys.Lett. B253, 138 (1991).

[16] P.Gornicki, Ann.Phys.(N.Y.) 202, 271 (1990).

[17] Yu.A.Sitenko and D.G.Rakityansky, Ukrainian Journ.Phys. 41, 329 (1996); Phys.Atom.Nucl. 60, $247 ; 258$ (1997).

[18] Yu.A.Sitenko, Phys.Lett. B387, 334 (1996)

[19] Yu.A.Sitenko, Phys.Atom.Nucl. 60, 2102 (1997); (E) 62, 1084 (1999).

[20] Yu.A.Sitenko and D.G.Rakityansky, Phys.Atom.Nucl. 60, 1497 (1997); Ukrainian Journ.Phys. 43, 761 (1998).

[21] Yu.A.Sitenko, Phys.Atom.Nucl. 62, 1056 (1999).

[22] A.J.Niemi and G.W.Semenoff, Phys.Rev.Lett. 51, 2077 (1983).

[23] L.Alvarez-Gaumé and E.Witten, Nucl.Phys. B234, 269 (1983).

[24] A.N.Redlich, Phys.Rev.Lett. 52, 18 (1984); Phys.Rev. D29, 2366 (1984).

[25] L.Alvarez-Gaumé, S.Della Pietra and G.Moore, Ann.Phys.(N.Y.) 163, 283 (1985).

[26] J.Weidmann, Math.Z. 119, 349 (1971).

[27] N.I.Akhiezer and I.M.Glazman, Theory of Linear Operators in Hilbert Space (Pitman, Boston, 1981) V.2.

[28] C.Itzykson and J.-B.Zuber, Quantum Field Theory (McGraw-Hill, New York, 1980).

[29] Yu.A.Sitenko and D.G.Rakityansky, Phys.Atom.Nucl. 61, 1497 (1998).

[30] J.Schwinger, Phys.Rev. 128, 2425 (1962).

[31] S.-S.Shei, Phys.Rev. D6, 3469 (1972).

[32] R.Jackiw, Dynamical symmetry breaking, in Laws of Hadronic Matter, edited by A.Zichichi (Academic Press, New York, 1975).

[33] P.Giacconi, S.Ouvry S. and R.Soldati, Phys.Rev. D50, 5358 (1994).

[34] A.Moroz, Phys.Lett. B358, 305 (1995).

[35] F.Wilczek, Phys.Rev.Lett. 48, 1144; 49, 957 (1982).

[36] R.Jackiw and A.N.Rellich, Phys.Rev.Lett. 50, 555 (1983).

[37] Y.-W.Chen, F.Wilczek, E.Witten and B.Halpern, Int.J.Mod.Phys. B3, 1001 (1989).

[38] P.A.M.Dirac, Proc.Roy.Soc.(London) A133, 60 (1931).

[39] M.G.Alford, J.March-Russel and F.Wilczek, Nucl.Phys. B328, 140 (1989) 
[40] T.W.Appelquist, M.Bowick, D.Karabali and L.C.R.Wijewardhana, Phys.Rev. D33, 3704 (1986).

[41] N.Dorey and N.E.Mavromatos, Nucl.Phys. B386, 614 (1992).

[42] V.P.Gusynin, V.A.Miransky and I.A.Shovkovy, Phys.Rev.Lett. 73, 3499 (1994); Phys.Rev. D52, 4718 (1995).

[43] G.Dunne and T.Hall, Phys.Rev. D53, 2220 (1996).

[44] Handbook of Mathematical Functions, edited by M.Abramowitz and I.A.Stegun (Dover, New York, 1972). 


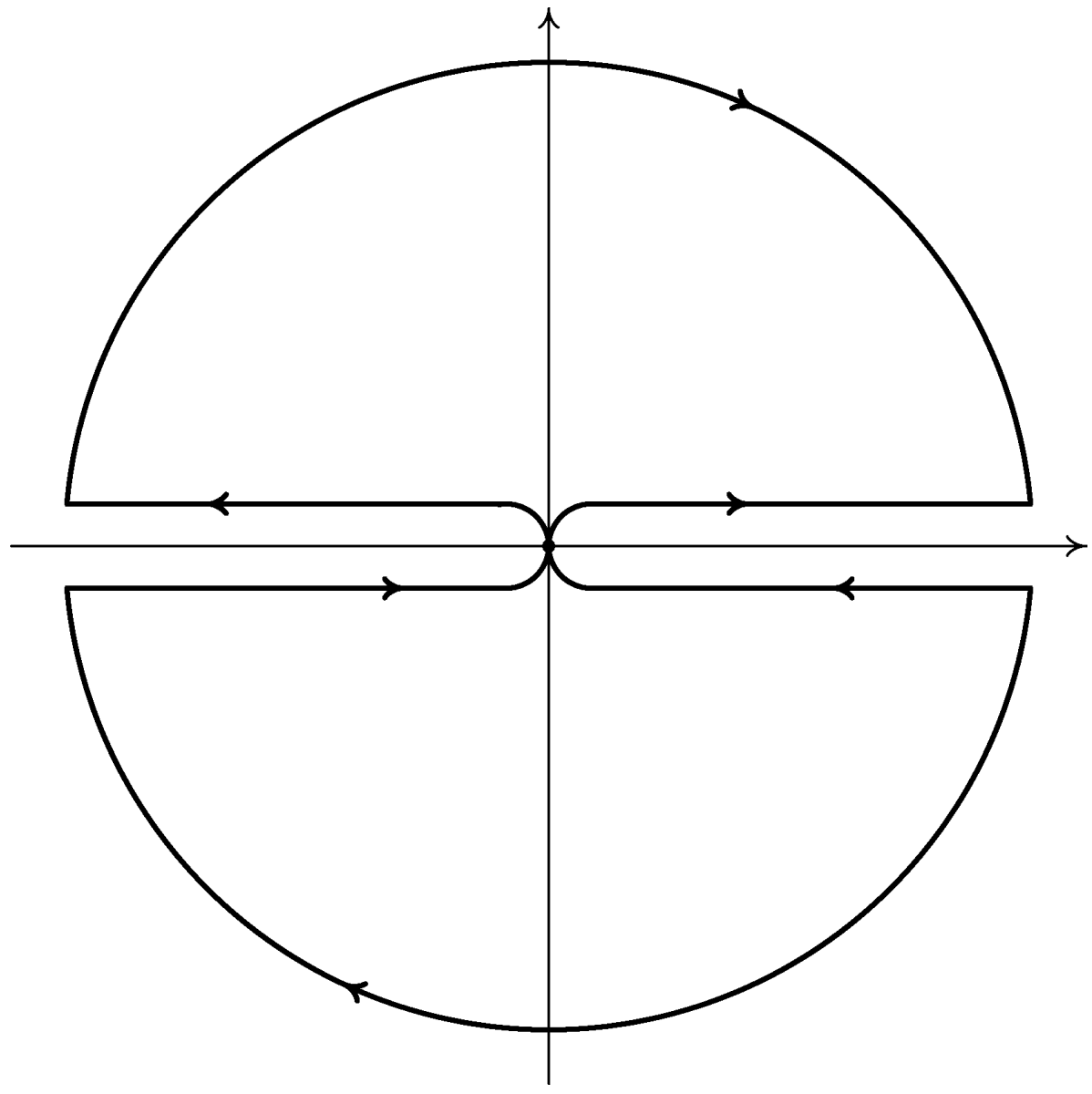

Fig. 1

Yu. A. Sitenko 


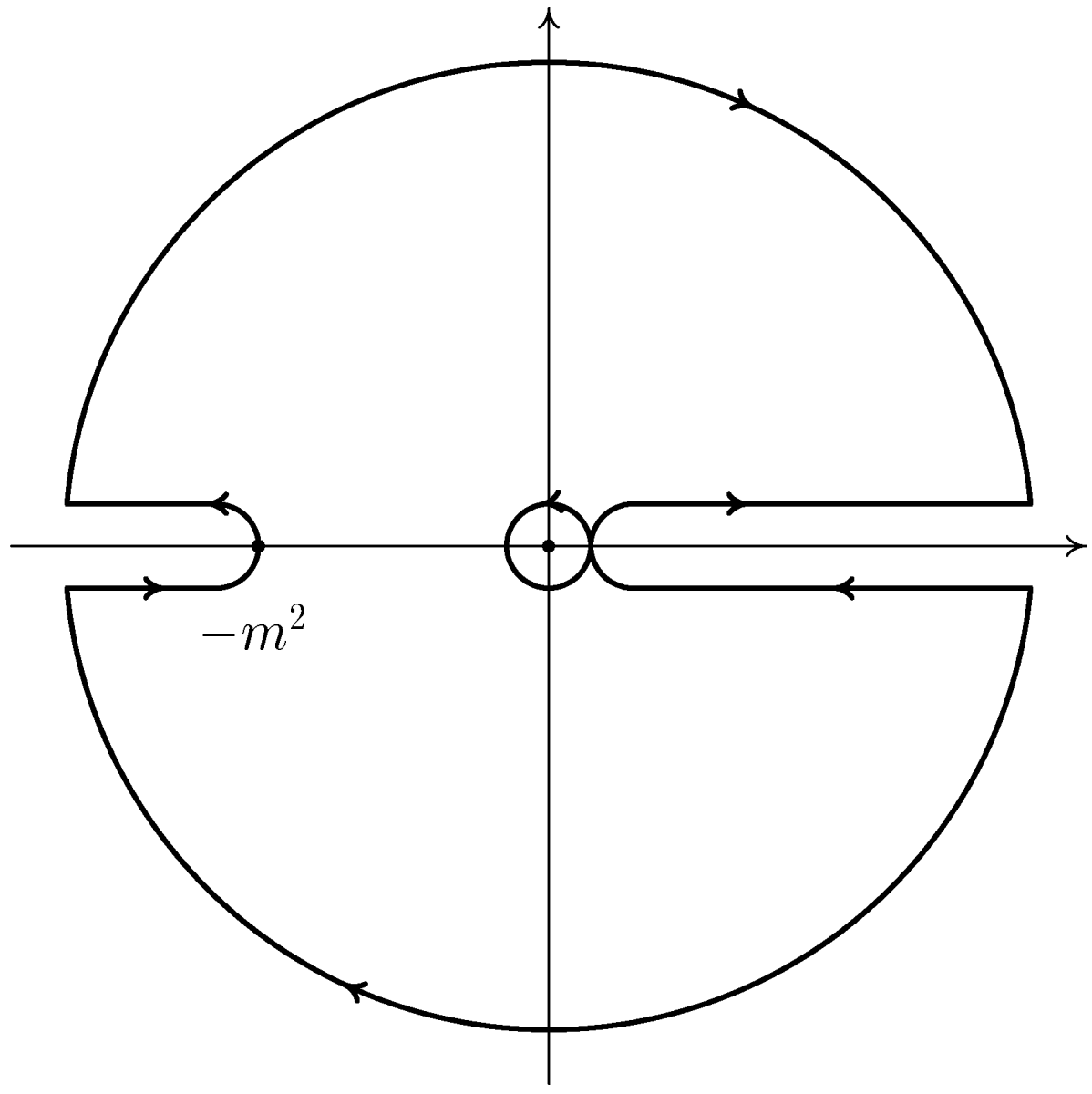

Fig. 2

Yu. A. Sitenko 


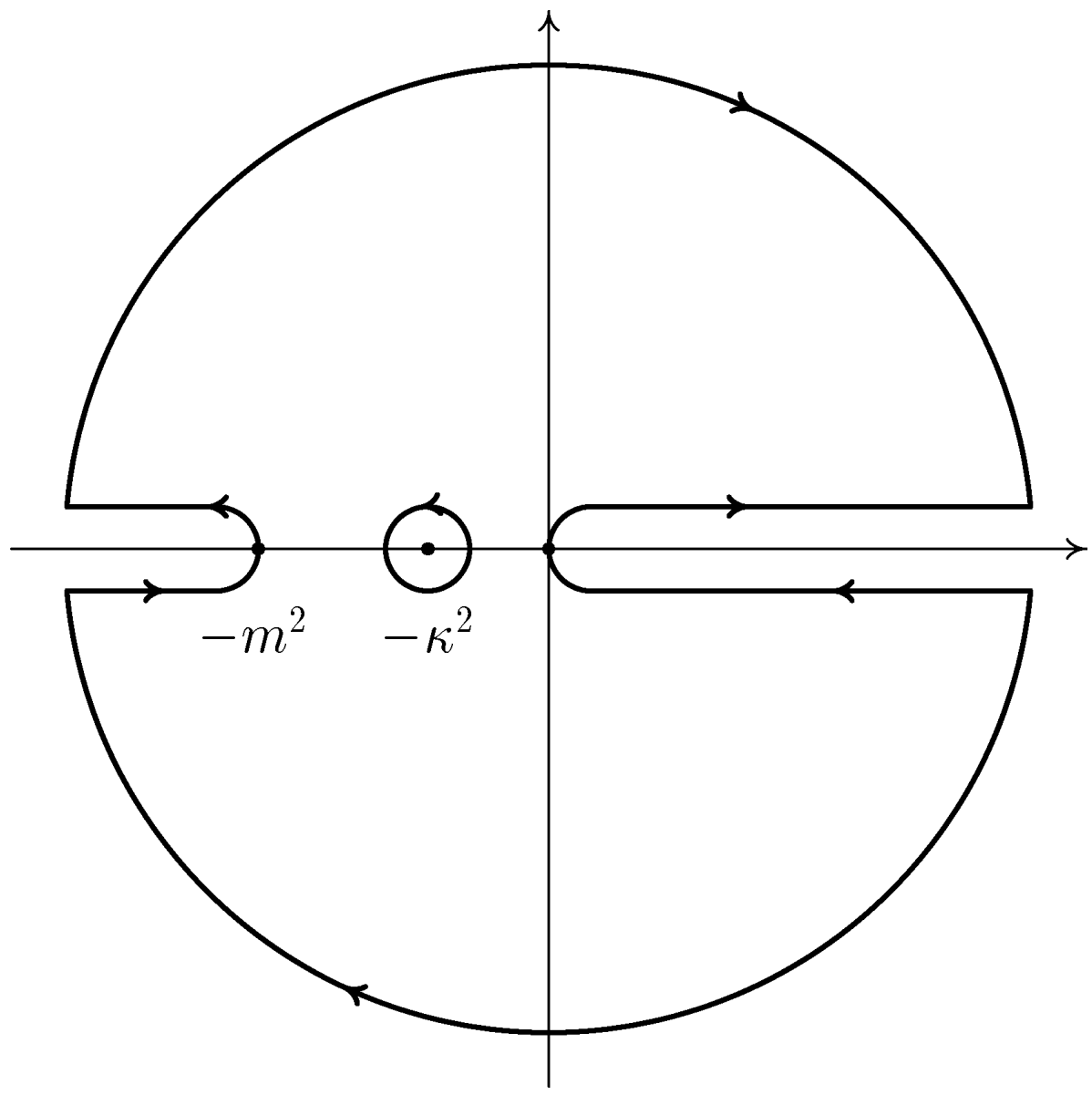

Fig. 3

Yu. A. Sitenko 


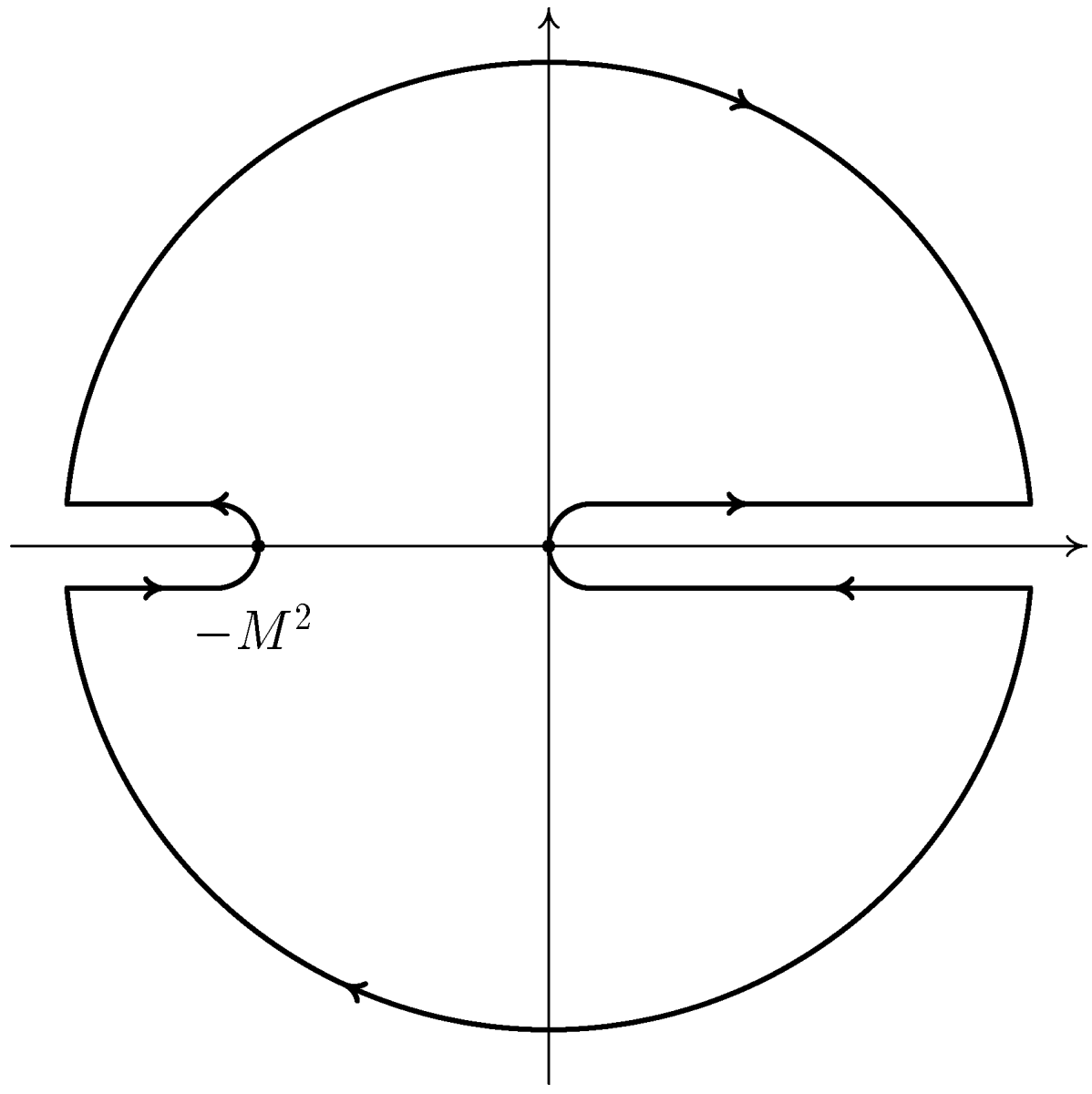

Fig. 4

Yu. A. Sitenko 